\title{
How Demography, Life History, and Kinship Shape the Evolution of Genomic Imprinting
}

\author{
Jeremy Van Cleve, ${ }^{\star}$ Marcus W. Feldman, and Laurent Lehmann ${ }^{\dagger}$ \\ Department of Biology, Stanford University, Stanford, California 94305 \\ Submitted April 4, 2010; Accepted June 30, 2010; Electronically published August 25, 2010 \\ Online enhancement:appendix.
}

ABSTRACT: How phenomena like helping, dispersal, or the sex ratio evolve depends critically on demographic and life-history factors. One phenotype that is of particular interest to biologists is genomic imprinting, which results in parent-of-origin-specific gene expression and thus deviates from the predictions of Mendel's rules. The most prominent explanation for the evolution of genomic imprinting, the kinship theory, originally specified that multiple paternity can cause the evolution of imprinting when offspring affect maternal resource provisioning. Most models of the kinship theory do not detail how population subdivision, demography, and life history affect the evolution of imprinting. In this work, we embed the classic kinship theory within an island model of population structure and allow for diverse demographic and life-history features to affect the direction of selection on imprinting. We find that population structure does not change how multiple paternity affects the evolution of imprinting under the classic kinship theory. However, if the degree of multiple paternity is not too large, we find that sex-specific migration and survival and generation overlap are the primary factors determining which allele is silenced. This indicates that imprinting can evolve purely as a result of sex-related asymmetries in the demographic structure or life history of a species.

Keywords: kinship theory, overlapping generations, sex-biased dispersal, dominance, sex-specific selection.

\section{Introduction}

Although Mendel's rules provide the conceptual bedrock for most population genetic and evolutionary theory, deviations from these rules are eagerly studied, as they often involve unusual phenotypes and pose novel evolutionary questions. One such deviation, genomic imprinting, has received a great deal of interest from both empirical and theoretical biologists (Bartolomei and Tilghman 1997;

* Corresponding author. Present address: Santa Fe Institute, 1399 Hyde Park Road, Santa Fe, New Mexico 87501; e-mail: vancleve@santafe.edu.

$\dagger$ Present address: Department of Biology, University of Neuchâtel, Rue EmileArgand 11, 2000 Neuchâtel, Switzerland.

Am. Nat. 2010. Vol. 176, pp. 000-000. (C) 2010 by The University of Chicago. 0003-0147/2010/17604-52062\$15.00. All rights reserved.

DOI: $10.1086 / 656277$
Wilkins and Haig 2003). In genomic imprinting, the expression level of a given allele is conditional on the parent of origin of that allele and typically involves the silencing of either the maternally or the paternally derived allele.

A number of theories have been developed to explain the evolution of genomic imprinting. One recent hypothesis suggests that imprinting can evolve in response to sexspecific selection or so-called sexual conflict (Day and Bonduriansky 2004; Van Cleve and Feldman 2007). In this scenario, alleles donated by the sex experiencing stronger selection against deleterious alleles are predicted to be expressed, while alleles donated by the other sex are silenced. Another recent theory suggests that expression of maternally derived alleles and silencing of paternally derived alleles evolved because of selection for coadaptation between maternal and offspring phenotypes (Wolf and Hager 2006).

The most prominent explanation for the evolution of genomic imprinting, however, is the kinship hypothesis (Haig 1992). This explanation is predicated on the assumption that offspring can influence the allocation of maternal resources postfertilization, which is the case for placental mammals and marsupials. The kinship hypothesis predicts that maternally derived copies of genes that increase the uptake of maternal resources will be silenced and paternally derived copies of the same gene will be expressed when relatedness between maternal gene lineages within offspring competing for maternal resources is higher than relatedness between paternal gene lineages. Such a relatedness asymmetry may be created when the offspring of a given female have multiple fathers. The pattern would then be expected to be reversed for genes that decrease maternal-resource uptake.

Previous analyses of the kinship hypothesis have assumed additive genetic effects and a family-structured population in which interactions among kin take place only between offspring of the same brood in an otherwise panmictic population (Haig 1992; Mochizuki et al. 1996; Spencer et al. 1998, 2004; Haig and Wilkins 2000; Green- 
Table 1: Variables and parameters in the model

\begin{tabular}{ll}
\hline Symbol & \multicolumn{1}{c}{ Definition } \\
\hline$N_{\mathrm{f}}\left(N_{\mathrm{m}}\right)$ & Number of adult females (males) in each deme \\
$N$ & Total population size in each deme: $N_{\mathrm{f}}+N_{\mathrm{m}}$ \\
$D$ & Number of demes \\
$\tau$ & Number of males with which a female mates, with replacement \\
$r$ & Sex ratio: $N_{\mathrm{m}} / N$ \\
$m\left(m_{\mathrm{f}}, m_{\mathrm{m}}\right)$ & Migration rate (for females and males) \\
$h$ & Dominance coefficient of the resident allele $a$ \\
$z$ & Level of expression from a resource-enhancer gene in a focal individual \\
$z_{a a}$ & Level of gene expression in an aa homozygote \\
$x_{a}\left(y_{a}\right)$ & Gene expression level from maternally (paternally) derived $a$ alleles \\
$\delta_{x}\left(\delta_{y}\right)$ & Deviations in gene expression level between alleles $A$ and $a$ for maternally (paternally) derived copies \\
$b(\bar{z})$ & Female fertility as a function of average offspring gene expression level $\bar{z}$ \\
$s_{\mathrm{f}}(z)\left(s_{\mathrm{m}}(z)\right)$ & Female (male) survival probabilities as a function of individual gene expression \\
$p_{\mathrm{fij}}, p_{\mathrm{fi}}, p_{\mathrm{f}}$ & Realized frequency of $A$ in female $j$ of deme $i$, average frequency of $A$ in females in deme $i$, and average \\
$\alpha_{\mathrm{f}}\left(\alpha_{\mathrm{m}}\right)$ & frequency of $A$ in females across all demes; frequencies in males are defined similarly \\
$Q_{u v}$ & Class reproductive value of females (males) \\
$S_{x}\left(z_{a a}\right)\left(S_{y}\left(z_{a a}\right)\right)$ & Probability that two alleles from different individuals of sex $u$ and $v$ sampled within a deme are identical by \\
$z_{a a}^{x}\left(z_{a a}^{y}\right)$ & Derivative of $\Delta p$ with respect to $\delta_{x}\left(\delta_{y}\right):$ selection gradient of $\delta_{x}\left(\delta_{y}\right)$ \\
& Maternally (paternally) derived expression levels that guarantee that $S_{x}\left(z_{a a}\right)=0\left(S_{y}\left(z_{a a}\right)=0\right) ;$ expression \\
\hline
\end{tabular}

wood-Lee et al. 2002; Mills and Moore 2004; Úbeda 2008). But many natural populations consist of local demes that are connected by dispersal (Lawson Handley and Perrin 2007). This spatial structure can have strong consequences for the evolution of populations (Wright 1931). In particular, gene frequency fluctuations induced by finite population size and limited migration (e.g., genetic drift) lead to the buildup of relatedness among neighbors, where asymmetries in genetical ties can be shaped by various lifehistory factors (e.g., Hamilton 1967; Charnov 1982; Frank 1998). These factors suggest that population structure (Haig 1997, 2000a; Spencer and Clark 2006) and lifehistory factors could have an important role in the evolution of genomic imprinting, for either the direction of imprinting or the level of imprinted gene expression.

Here, we address these issues and embed the key lifehistory and mating-system assumptions of the kinship hypothesis into a metapopulation system with an island model of population structure. In order to investigate the effect of demography on the evolution of imprinting under the kinship hypothesis, we construct a mathematical model based on a weak-selection approximation for the change in the frequency of a focal allele that causes a specified phenotypic effect (Rousset and Billiard 2000; Rousset 2004). This model allows us to study how finite population size, limited migration, biased sex ratios, sex-specific values of viability selection and migration, generation overlap, and allelic dominance all effect the prediction of the classical kinship hypothesis (Haig 1992); the classic prediction is that under multiple paternity, alleles that increase resource uptake should be expressed when paternally derived and silenced when maternally derived.

\section{Model Framework$$
\text { Life Cycle }
$$

We consider an island model of population structure (Wright 1931); there are $D$ demes (or patches), each equally connected to the others through migration. Although we formulate the model generically for a finite number of demes, we will analyze the model in the limit as the number of demes becomes very large $(D \rightarrow \infty)$. Each deme is assumed to contain $N$ diploid individuals, $N_{\mathrm{f}}=$ $(1-r) N$ females and $N_{\mathrm{m}}=r N$ males, where $r$ is the fraction of males. Table 1 contains a full list of the model parameters and variables.

The life cycle of the individuals begins with random mating among adult males and females. A very large number of juvenile males and females are produced, and they suffer density-independent mortality during their development into adults; we assume that this mortality depends on the phenotypes of individuals and their sex. Juveniles who survive then migrate independently of each other to a different deme with probability $m_{\mathrm{f}}$ for females and $m_{\mathrm{m}}$ for males. During each iteration of the life cycle, adults who currently reside in the deme suffer mortality according to their phenotype and sex. Finally, as juveniles mature 
into adults, they compete for the open patches vacated by dying adults. Density-dependent effects at this stage maintain the male and female adult populations at constant sizes $N_{\mathrm{f}}$ and $N_{\mathrm{m}}$, respectively.

The classic kinship hypothesis (Haig 1992, 1997) assumes that females have an opportunity to mate with more than one male. Here, we use the simplest such mating scheme and assume that each female randomly chooses $\tau$ males with replacement from the $N_{\mathrm{m}}$ males in her deme; this mating system can be classified as a type of polygynandry. Thus, $\tau$ can be thought of as a measure of how many males sire offspring in a brood, or as the degree of multiple paternity.

Following classical analyses of the kinship hypothesis (Haig 1992, 1997; Mochizuki et al. 1996), the phenotype we focus on is the level of expression of a resourceenhancer (Úbeda 2008) gene that increases the rate of uptake of maternal resources by offspring during development. In order to track the evolutionary dynamics of this phenotype, we develop a population genetic model using the weak-selection approach developed by Rousset and colleagues (e.g., Rousset and Billiard 2000; Rousset 2004; Roze and Rousset 2004, 2008) for the study of evolution in subdivided populations.

\section{Genotypes and Phenotypes}

We assume that a mutant allele, denoted $A$, and a wildtype allele, denoted $a$, segregate at the locus controlling the level of gene expression. Since we are interested in exploring the possibility that expression levels may differ according to the sex of the parent who donated the allele, we write the phenotype of an individual homozygous for the resident allele as $z_{a a}=x_{a}+y_{a}$, where $x_{a}$ is the gene expression level of $a$ when maternally derived and $y_{a}$ is the level when paternally derived (both $x_{a}$ and $y_{a}$ take only positive values). The phenotype of an individual homozygous for the mutant allele is assumed to be given by $z_{A A}=x_{a}+y_{a}+\delta_{x}+\delta_{y}$, where the parameters $\delta_{x}$ and $\delta_{y}$ measure, respectively, how much the maternally derived and paternally derived expression levels of $A$ deviate from those of $a$ and can take both positive or negative values, as long as the resulting expression levels of $A$ are nonnegative. Finally, we allow dominance interactions between the levels of paternal and maternal gene expression so that the phenotype of heterozygote individuals having received $A$ (or $a$ ) maternally is assumed be to be given by $z_{A a}=$ $x_{a}+y_{a}+2(1-h) \delta_{x}\left(\right.$ or $\left.z_{a A}=x_{a}+y_{a}+2(1-h) \delta_{y}\right)$, where $h$ may be thought of as measuring the level of penetrance of the expression level induced by the $a$ allele. When $h=1$, the mutant allele has no effect on expression level, and the expression levels from both maternally and paternally derived copies are fully affected when $h=0$.
It will be convenient for our analysis to introduce the allele frequency variable $p_{u f i j}\left(p_{u m i j}\right)$, which is equal to 1 when the maternally (paternally) derived allele in focal individual $j$ of sex $u(u=\mathrm{m}$ or $\mathrm{f})$ in deme $i$ is $A$ and 0 otherwise. With this, we can write the phenotype of individual $j$ of sex $u$ in deme $i$ as

$$
\begin{aligned}
z\left(p_{u f i j}, p_{u \mathrm{mij}}\right)= & p_{u f i j} p_{u \mathrm{~m} i j}\left(x_{a}+y_{a}+\delta_{x}+\delta_{y}\right) \\
& +p_{u \mathrm{fij}}\left(1-p_{u \mathrm{~m} i j}\right)\left[x_{a}+y_{a}+2(1-h) \delta_{x}\right] \\
& +\left(1-p_{u \mathrm{fij}}\right) p_{u \mathrm{mi} i j}\left[x_{a}+y_{a}+2(1-h) \delta_{y}\right] \\
& +\left(1-p_{u \mathrm{fi} i}\right)\left(1-p_{u \mathrm{mij} i j}\right)\left(x_{a}+y_{a}\right) .
\end{aligned}
$$

The crucial life-history assumption in the kinship hypothesis is that the phenotype $z$ of an offspring affects both the survival of the offspring itself and the total maternal fertility as a result of differential consumption of maternal resources during development (Haig 1992). Since females have a finite amount of resources to devote to offspring development, a high rate of consumption of maternal resources by each offspring during development results in a smaller brood size; that is, there is a trade-off between offspring survival and maternal fertility. In order to formalize this trade-off, we let the probability $s_{\mathrm{f}}(z)$ (or $s_{\mathrm{m}}(z)$ ) that a given juvenile female (or male) survives density-independent selection be an increasing function of its phenotype $z$, where the shape of the function expresses diminishing return. In an extension of the kinship hypothesis, we assume that adult phenotype also affects survival to the next generation; for simplicity, an adult female (or male) survives with the same probability as a juvenile, $s_{\mathrm{f}}(z)\left(\right.$ or $\left.s_{\mathrm{m}}(z)\right)$. In the second part of the tradeoff, we suppose that the fertility $b(\bar{z})$ of a focal adult female is a decreasing function of the average gene expression level $\bar{z}$ among her offspring. During mating, females mate with $\tau$ males, with replacement as described above, and produce a single brood from the stored sperm of those matings. A large number of zygotes are produced from those sperm and grow to the juvenile stage.

\section{Change of Allele Frequency}

The fitness of a focal adult individual is defined as the expected number of offspring that survive to adulthood. Since we allow for sex-specific survival and a flexible sex ratio, we must account for fitness in a sex-specific way. Let $W_{u i j}$ be the expected number of adult offspring of sex $v$ produced by adult $j$ of sex $u$ in deme $i$. Then, there are four fitness functions: $W_{\text {ffij }}, W_{\text {fmiji, }}, W_{\text {mfij }}$ and $W_{\text {mmiji }}$. Using these fitness functions, we can write the expected change in the frequency of allele $A$ over a single generation, $\Delta p$, as 


$$
\begin{aligned}
\Delta p= & \frac{\alpha_{\mathrm{f}}}{2 D} \sum_{i=1}^{D}\left(\frac{1}{N_{\mathrm{f}}} \sum_{j=1}^{N_{\mathrm{f}}} W_{\mathrm{ffi} i j} p_{\mathrm{f} i j}+\frac{1}{N_{\mathrm{m}}} \sum_{j=1}^{N_{\mathrm{m}}} W_{\mathrm{mfij}} p_{\mathrm{m} i j}\right) \\
& +\frac{\alpha_{\mathrm{m}}}{2 D} \sum_{i=1}^{D}\left(\frac{1}{N_{\mathrm{f}}} \sum_{j=1}^{N_{\mathrm{f}}} W_{\mathrm{fm} i j} p_{\mathrm{fij}}+\frac{1}{N_{\mathrm{m}}} \sum_{j=1}^{N_{\mathrm{m}}} W_{\mathrm{mm} i j} p_{\mathrm{m} i j}\right)-p,
\end{aligned}
$$

where $p=\alpha_{\mathrm{f}} p_{\mathrm{f}}+\alpha_{\mathrm{m}} p_{\mathrm{m}}$ is the reproductive valueweighted average of the frequency of $A$ in all females in all demes, $p_{\mathrm{f}}$, and all males in all demes, $p_{\mathrm{m}}$, with $\alpha_{\mathrm{f}}$ and $\alpha_{\mathrm{m}}$ being the class reproductive values of males and females, respectively (Taylor 1990); $p_{\mathrm{fij}}$ is the realized frequency of $A$ in female $j$ in deme $i$ in the current generation and can take the values $0,1 / 2$, or $1 ; p_{\mathrm{m} i j}$ is defined similarly for male $j$. The fitness functions depend on full genotype frequencies, but these can be expressed as functions of the frequency variables $p_{\mathrm{fij}}$ and $p_{\mathrm{mij}}$, which are illustrated below. The factor of $1 / 2$ in equation (2) accounts for the frequency of transmission of genes from parent to offspring due to meiosis.

\section{Analysis: Weak-Selection Approximation}

\section{Fitness Function}

In order to evaluate a weak-selection approximation to $\Delta p$, we build a first-order Taylor expansion of $\Delta p$ about $\delta_{x}=0$ and $\delta_{y}=0$. Since fitness is a function of phenotype, assuming that $\delta_{x}$ and $\delta_{y}$ are small entails that selection is weak (Wild and Traulsen 2007). In order to calculate the weak-selection approximation to $\Delta p$, we need firstorder approximations of the four fitness functions. As is commonly found in island models (e.g., Roze and Rousset 2004; Lehmann et al. 2007; Rousset and Roze 2007), we can approximate the expected number of adult offspring of $\operatorname{sex} v$ left by individual $j$ of sex $u$ in deme $i$ when the number of demes grows large as

$$
W_{u v i j} \approx 2 \varepsilon_{u v} S_{u i j}+\frac{\left(1-S_{v i}\right)\left(1-m_{v}\right) J_{u v i j}}{\left(1-m_{v}\right) J_{u v i}+m_{v} J_{u v}}+\frac{\left(1-S_{v}\right) m_{v} J_{u v i j}}{J_{u v}}
$$

(see "Fitness Functions" in the online edition of the American Naturalist), where $\varepsilon_{u v}$ is equal to 1 when parent and offspring are of the same sex (i.e., $v=u$ ) and to 0 when they are of opposite sexes (i.e., $v \neq u$ ). The probability that adult $j$ of sex $u$ living in deme $i$ survives is given by $S_{u i j}=s_{u}\left(z_{u i j}\right)$, where the phenotype $z_{u i j}$ is a function of the mutant allele frequency $(0,1 / 2$, or 1$)$ in individual $j$. The average of $S_{u i j}$ over all individuals of sex $u$ in deme $i$ is given by $S_{u i}$, and the average over all demes is given by $S_{u}$. The term $J_{u v i j}$ is the expected number of juveniles of sex $v$ produced by individual $j$ of sex $u$ in deme $i$ that survive to the migration stage. Averaging over all individuals of sex $u$ in the deme yields $J_{u v i}$, and averaging over all demes yields $J_{u v}$. The factor of 2 for the survival probability of the focal adult in equation (3), $S_{u i j}$, accounts for the fact that an adult that survives to the next generation "inherits" both of its alleles in the next generation, whereas its offspring inherit only one because of meiosis.

\section{Fertility}

In simple cases where mating is random and the phenotype under consideration has an effect only on survival or fertility, the expected number of juveniles produced by each sex that survive to the migration stage becomes a simple function of the phenotype of a focal individual and the average phenotype in a focal deme (Rousset 2004). However, our model assumes that there is a trade-off between female fertility and offspring survival, so evaluating these expectations requires a careful accounting of how the mating system operates and how offspring survival and female fertility interact as a result of their shared genetic basis. In "Expected Number of Juveniles before Migration" in the online edition of the American Naturalist, we show that, despite this complexity, a first-order expression for the expected number of juveniles is still a function of the phenotype of the focal individual and the focal deme. For example, the expected number of juveniles of sex $v$ produced by female $j$ in deme $i$ can be expressed as

$$
J_{\mathrm{f} v i j}=b\left(z\left(p_{\mathrm{fij}}, p_{\mathrm{m} i}\right)\right) s_{v}\left(z\left(p_{\mathrm{fij}}, p_{\mathrm{m} i}\right)\right)+O\left(\delta^{2}\right),
$$

which is simply the product of female fertility and offspring survival where $z\left(p_{\mathrm{fi} i}, p_{\mathrm{m} i}\right)$ is the average gene expression in offspring of a focal female and $\delta$ is the larger of the two phenotypic deviations $\delta_{x}$ and $\delta_{y}$. Since we assume that females choose males randomly with respect to their genotype, the average gene expression in the focal female's offspring is a function of her allele frequency, $p_{\mathrm{fij}}$, and the average allele frequency in males in the focal deme $i, p_{\mathrm{m} i}$.

The expected number of juveniles of sex $v$ produced by a focal male is more complex because of female control of reproduction and because females mate $\tau$ times:

$$
\begin{aligned}
J_{\mathrm{m} v i j}= & \frac{N_{\mathrm{f}}}{N_{\mathrm{m}}} b\left(\frac{1}{\tau} z\left(p_{\mathrm{fi}}, p_{\mathrm{m} i j}\right)+\left(1-\frac{1}{\tau} z\left(p_{\mathrm{fi}}, p_{\mathrm{m} i}\right)\right)\right) \\
& \times s_{v}\left(z\left(p_{\mathrm{f} i}, p_{\mathrm{m} i j}\right)\right)+O\left(\delta^{2}\right) .
\end{aligned}
$$

Here, the fertility of a focal male is determined by the 
average gene expression in all offspring of all females with which the male mates. This expression level, given by the argument to the fertility function $b$ in equation (5), is the sum of the average gene expression in offspring of the focal male, $z\left(p_{\mathrm{f} i}, p_{\mathrm{m} i j}\right)$, weighted by the average fraction of paternity the focal male has in each clutch of offspring, $1 / \tau$, and the average expression level in all offspring of all males (and females) in the deme, $z\left(p_{\mathrm{fi}}, p_{\mathrm{m} i}\right)$, weighted by the remaining paternity, $1-1 / \tau$, where $p_{\mathrm{f} i}$ is the average allele frequency in females in the focal deme $i$. The fraction $N_{\mathrm{f}} / N_{\mathrm{m}}$ in equation (5) reflects the effect of the sex ratio on absolute male fertility.

\section{Selection Gradient}

Using the definition of gene expression level $z$ in equation (1) and the number of juveniles produced by females and males in equations (4) and (5), respectively, we can calculate first-order expressions for the fitness function given by equation (3), as is usually carried out with the direct fitness method (Taylor and Frank 1996; Rousset 2004). Inserting these functions into the formula for $\Delta p$ given in equation (2), we arrive at

$$
\Delta p=p(1-p)\left(\mathcal{S}_{x} \delta_{x}+\mathcal{S}_{y} \delta_{y}\right)+O\left(\delta^{2}\right)
$$

(see "Overlapping Generations" in the online edition of the American Naturalist), where $p(1-p)$ is the variance in gene frequency in an individual, $\mathcal{S}_{y}$ is the selection gradient on the mutant allele resulting from its expression when paternally inherited, and $\mathcal{S}_{x}$ is the selection gradient on the mutant allele when maternally inherited. These two selection gradients are given by

$$
\begin{aligned}
\mathcal{S}_{x}= & \alpha_{\mathrm{f}} s_{\mathrm{f}} B_{\mathrm{f}} R_{a s, \mathrm{ff}}+\alpha_{\mathrm{m}} s_{\mathrm{m}} B_{\mathrm{m}} R_{a s, \mathrm{mf}} \\
& +\beta\left[B_{\mathrm{f}} R_{j s, \mathrm{ff}}+B_{\mathrm{m}} R_{j s, \mathrm{mf}}-C\left(R_{b, \mathrm{ff}}+R_{b, \mathrm{mf}}\right)\right]
\end{aligned}
$$

and

$$
\begin{aligned}
\mathcal{S}_{y}= & \alpha_{\mathrm{f}} \mathcal{s}_{\mathrm{f}} B_{\mathrm{f}} R_{a s, \mathrm{fm}}+\alpha_{\mathrm{m}} s_{\mathrm{m}} B_{\mathrm{m}} R_{a s, \mathrm{~mm}} \\
& +\beta\left[B_{\mathrm{f}} R_{j s, \mathrm{fm}}+B_{\mathrm{m}} R_{j s, \mathrm{~mm}}-C\left(R_{b, \mathrm{fm}}+R_{b, \mathrm{~mm}}\right)\right],
\end{aligned}
$$

where $s_{u}$ is the probability that an adult of sex $u$ with the resident phenotype survives to the next generation, $\alpha_{\mathrm{f}}=\left(1-s_{\mathrm{m}}\right) /\left(2-s_{\mathrm{f}}-s_{\mathrm{m}}\right)$ and $\alpha_{\mathrm{m}}=\left(1-s_{\mathrm{f}}\right) /\left(2-s_{\mathrm{f}}-\right.$ $\left.s_{\mathrm{m}}\right)$ are the female and male reproductive values, respectively, and $\beta=\alpha_{\mathrm{f}}\left(1-s_{\mathrm{f}}\right)=\alpha_{\mathrm{m}}\left(1-s_{\mathrm{m}}\right)$. The functions $B_{u}=s_{u}^{\prime} / s_{u}$ in the selection gradients (a prime denotes a derivative) can be interpreted as the direct survival benefits to focal offspring of sex $u$ stemming from increase in its gene expression level, and $C=-b^{\prime} / b$ is the associated indirect fertility cost to a parent stemming from the focal offspring raising gene expression level.

The coefficients $R_{a s, u v}, R_{j s, u v}$, and $R_{b, u v}$ can be interpreted as imprinted measures of relatedness between different classes of individuals based on the effect of the mutant allele on adult survival $\left(R_{a s, u v}\right)$, juvenile survival $\left(R_{j s, u v}\right)$, and fertility $\left(R_{b, u v}\right)$, where the subscripts $u$ and $v$ specify the class of the actor. Recipients of fitness effects are adults because we measure fitness at the adult stage. For example, $R_{j s, \mathrm{mf}}$ can be interpreted as the relatedness value between a maternally derived allele $(\mathrm{f})$ in a male $(\mathrm{m})$ focal juvenile, the actor, and an allele in one of its own parents, where male and female parents are sampled with equal probability because of fair meiosis. Since the fertility of a female depends on the genotype of all offspring in her clutch, $R_{b, \mathrm{ff}}$ can be interpreted as the relatedness between a maternally derived allele (f) in a focal female (f) juvenile and a random allele in one of the parents of the clutch. Interpretations for the other relatedness coefficients are analogous.

\section{Scaled Relatedness}

Importantly, the coefficients $R_{a s, u v}, R_{j s, u v}$, and $R_{b, u v}$ are scaled measures of relatedness where the effect of local competition (sometimes referred to as kin competition) on the selection gradient is taken into account (Queller 1994). Hence, $R_{a s, u v}, R_{j s, u v}$, and $R_{b, u v}$ depend, as in previous models for subdivided populations (e.g., Taylor 1992; Roze and Rousset 2004; Lehmann et al. 2007; Rousset and Roze 2007), on various coefficients of probabilities of identity by descent (IBD) between sets of genes and on the parameters of the model (see eqq. [12], [14], [19], and [22]). The evaluation of these probabilities of identity is carried out in "Probabilities of Identity by Descent" in the online edition of the American Naturalist. However, without fully evaluating $R_{a s, u v}, R_{j s, u v}$, and $R_{b, u v}$, we can infer from equations (6) and (A21)-(A23) in the online edition of the American Naturalist how female fertility, offspring survival, and allelic dominance will affect the change in mutant frequency.

\section{Dominance}

The degree of dominance, or penetrance, of the allele $a$, namely, the coefficient $h$, affects $\Delta p$ by adding geneticidentity terms in the $R_{j s, u v}$ and $R_{b, u v}$ coefficients in equations (A21)-(A23) that contain products of three allele frequencies. These terms are scaled by $1-2(1-h)$ so that the dominance terms augment $\Delta p$ when $h<1 / 2$, and decrease $\Delta p$ when $h>1 / 2$. The dominance terms do not depend on whether the focal allele is maternally or paternally derived, which means that the dominance terms 
affect both gene expression level deviations, $\delta_{x}$ and $\delta_{y}$, identically. In contrast, the "additive" terms that do not disappear when $h=1 / 2$ scale $\delta_{x}$ and $\delta_{y}$ independently. In order for selection to increase the degree of imprinting through an increase in the frequency of the allele $A$, the expression-level deviations $\delta_{x}$ and $\delta_{y}$ must have independent effects on $\Delta p$. Thus, dominance cannot by itself select for imprinting; rather, dominance can exert selection on the total expression level of both maternally and paternally derived alleles and in doing so may affect the strength of selection for imprinting. We examine this effect of dominance explicitly in "The Effect of Dominance on $\Delta p$ " in the online edition of the American Naturalist. In the rest of the article, we assume that $h=1 / 2$.

\section{Direction of Selection on Imprinting and Evolutionary Stability}

Under additive gene action and our weak-selection approximation, the selection gradients, $\mathcal{S}_{x}$ and $\mathcal{S}_{y}$ are frequency independent. This lack of frequency dependence allows us to derive candidate evolutionarily stable (ES) values of $x_{a}$ and $y_{a}$, since a newly arisen mutant $A$ will either invade and increase in frequency to fixation or will become extinct. We denote the candidate ES values of $x_{a}$ and $y_{a} x_{a}^{*}$ and $y_{a}^{*}$, respectively. In order to find $x_{a}^{*}$ and $y_{a}^{*}$, we define $z_{a a}^{x}$ and $z_{a a}^{y}$ to be nonzero solutions of

$$
\mathcal{S}_{x}\left(z_{a a}^{x}\right)=0
$$

and

$$
\mathcal{S}_{y}\left(z_{a a}^{y}\right)=0,
$$

respectively. If a maternally inherited allele completely determines the total expression level, then $z_{a a}^{x}$ can be seen as a candidate ES level of expression from the perspective of maternally derived expression; $z_{a a}^{y}$ can be seen as the analogous quantity from the perspective of paternally derived expression.

In order for a phenotype to evolve to a candidate ES value, mutant alleles may invade the population and reach fixation only when they bring the phenotype in the population closer to the ES value. This property, convergence stability, can be expressed mathematically as $\left.\left(\partial S_{x} / \partial z_{a a}\right)\right|_{z_{a a}=z_{a a}^{x}}<0$ and $\left.\left(\partial S_{y} / \partial z_{a a}\right)\right|_{z_{a a}=z_{a a}^{y}}<0$ for the candidate ES values $z_{a a}^{x}$ and $z_{a a}^{y}$, respectively, and is a necessary condition for long-term evolutionary stability (Eshel 1983). In general, we will assume that all candidate ES phenotypes are also convergence stable.

When the selection gradients are equal, $\mathcal{S}_{x}=\mathcal{S}_{y}$, $z_{a a}^{x}=z_{a a}^{y}$ and the candidate ES gene expression levels are the same for maternally and paternally derived alleles. In this case, any $x_{a}^{*}$ and $y_{a}^{*}$ that sum to $z_{a a}^{x}=z_{a a}^{y}$ are ES. This means that selection is indifferent to whether the total amount of expression is generated by Mendelian $\left(x_{a}^{*}=\right.$ $\left.y_{a}^{*}\right)$ or imprinted $\left(x_{a}^{*} \neq y_{a}^{*}\right)$ gene expression.

When the selection gradients $\mathcal{S}_{y}$ and $\mathcal{S}_{x}$ are not equal and the maternally and paternally derived gene expression levels have different candidate ES values, no values of $x_{a}^{*}$ and $y_{a}^{*}$ can simultaneously sum to both $z_{a a}^{x}$ and $z_{a a}^{y}$. As figure 1 shows, this results in silencing of one of the alleles and expression from the other allele at its maximal possible value, either $z_{a a}^{x}$ for $x_{a}^{*}$ or $z_{a a}^{y}$ for $y_{a}^{*}$ (Greenwood-Lee et al. 2002 provide an alternative dynamical explanation of this process). Which allele is silenced is determined by whether $z_{a a}^{y}$ is greater or less than $z_{a a}^{x}$. In other words, which allele is imprinted depends on whether $\mathcal{S}_{y}$ is greater or less than $\mathcal{S}_{x}$ (at the point where the first of the two selection gradients vanishes).

In order to understand this result, suppose that $\mathcal{S}_{y}>$ $\mathcal{S}_{x}$ and note that when $x_{a}$ and $y_{a}$ entail a gene expression level that is less than $z_{a a}^{x}, \mathcal{S}_{x}\left(z_{a a}\right)$ and $\mathcal{S}_{y}\left(z_{a a}\right)$ are positive and equation (6) shows that $A$ increases in frequency when it increases both maternally and paternally derived expression levels. A series of mutant-allele invasions and fixations leads $x_{a}$ and $y_{a}$ to approach $z_{a a}^{x}$. When $x_{a}+$

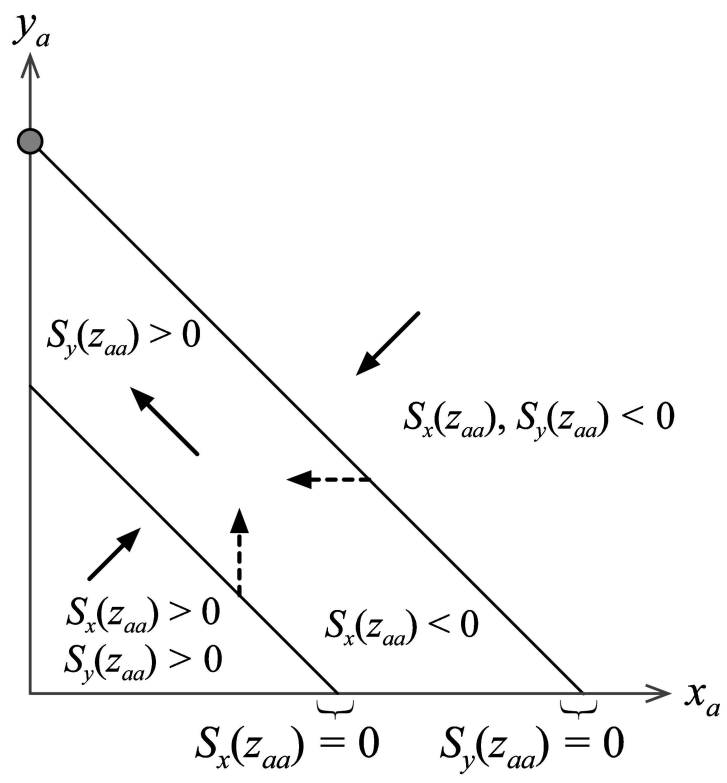

Figure 1: Convergence stability of $y_{a}^{*}=z_{a a}^{y}$ and $x_{a}^{*}=0$, denoted by the circle, when $h=1 / 2$ and $\tau>1$. The lower diagonal line indicates all $x_{a}$ and $y_{a}$ that solve $\mathcal{S}_{x}\left(z_{a a}\right)=0$, and the upper diagonal line is defined similarly for $\mathcal{S}_{y}\left(z_{a a}\right)=0$. The arrows indicate the general direction that a vector of $\delta_{x}$ and $\delta_{y}$ must have so that $\Delta p>0$. Given that $\partial \mathcal{S}_{x} / \partial z_{a a}<0$ and $\partial \mathcal{S}_{y} / \partial z_{a a}<0$, this leads to the evolutionarily stable values $y_{a}^{*}=z_{a a}^{y}$ and $x_{a}=0$. 
$y_{a}=z_{a a}^{x}$ allele $A$ increases in frequency if it increases expression from the paternal allele, $\delta_{y}>0$. Once the maternally and paternally derived expression levels evolve so that they are both less than $z_{a a}^{y}$ and greater than $z_{a a}^{x}$ allele $A$ invades the population when it induces decreased expression from the maternally derived allele and increased expression from the paternally derived allele. This leads to silencing of the maternally derived allele, $x_{a}^{*}=0$, and $y_{a}^{*}=z_{a a}^{y}$ (see fig. 1). Similar reasoning shows that the ES expression levels $x_{a}^{*}=0$ and $y_{a}^{*}=z_{a a}^{y}$ are approached when $\mathcal{S}_{x}>\mathcal{S}_{y}$. We show below how various combinations of life-history factors can lead the selection gradients $\mathcal{S}_{y}$ and $\mathcal{S}_{x}$ to differ and thus lead to the evolution of genomic imprinting.

\section{Results}

\section{Multiple Paternity: Direction of Imprinting}

We begin by analyzing the case with equal migration rates in the sexes $\left(m_{\mathrm{f}}=m_{\mathrm{m}}=m\right)$ and no generation overlap $\left(s_{\mathrm{f}}=s_{\mathrm{m}}=0\right.$ for adults). In this case, the selection gradients in equations (7) and (8) simplify so that $R_{j s, f u}=$ $R_{j, \mathrm{~m} u}=R_{j, u}$ and $R_{b, \mathrm{fu}}=R_{b, \mathrm{~m} u}=R_{b, u}$ and terms with $R_{a s, u v}$ disappear, which gives the selection gradients on the mutant allele as

$$
\begin{aligned}
& \mathcal{S}_{x}=\frac{1}{2}\left(B_{\mathrm{f}}+B_{\mathrm{m}}\right) R_{j s, \mathrm{f}}-C R_{b, \mathrm{f}}, \\
& \mathcal{S}_{y}=\frac{1}{2}\left(B_{\mathrm{f}}+B_{\mathrm{m}}\right) R_{j s, \mathrm{~m}}-C R_{b, \mathrm{~m}} .
\end{aligned}
$$

Substituting the probabilities of genetic identity from "Probabilities of Identity by Descent" into the scaledrelatedness coefficients weighting the benefits in equation (11) yields

$$
\begin{aligned}
R_{j s, u}= & \frac{1}{2}\left(\frac{1+Q_{\mathrm{fm}}}{2}+Q_{\mathrm{fm}}\right)-\frac{(1-m)^{2}}{2} \\
& \times\left[\frac{1}{N_{u}}\left(\frac{1+Q_{\mathrm{fm}}}{2}+Q_{\mathrm{fm}}\right)+\left(1-\frac{1}{N_{u}}\right)\left(Q_{u u}+Q_{\mathrm{fm}}\right)\right]
\end{aligned}
$$

where $Q_{u v}$ denotes the probability that two alleles in different adults, one sampled from sex $u$ and one from sex $v$, are identical by descent.

Equation (12) accounts for two different types of inclusive fitness effects accruing to the mutant gene lineage from increasing the gene expression of an allele donated by a parent of sex $u$ in a focal juvenile (the actor). The first fitness effect is a benefit to the parents of the focal juvenile (recipient) that stems from the juvenile having a higher survival probability. This is accounted for by the first term in equation (12), $\left(1+Q_{\mathrm{fm}}\right) / 4+Q_{\mathrm{fm}} / 2$, namely, the probability of identity between a focal allele sampled in the focal juvenile, which is derived from a parent of sex $u$, and an allele randomly sampled from a parent of the focal juvenile. The parent that has contributed the focal allele to the juvenile is sampled with probability $1 / 2$ (meiosis is fair), and the probability of identity between the two alleles is then $\left(1+Q_{\mathrm{fm}}\right) / 2$, where $Q_{\mathrm{fm}}$ has to be understood as the inbreeding coefficient (at equilibrium, the inbreeding coefficient is equivalent to the probability of identity between two alleles, one randomly sampled in an adult male and the other in a female; Gandon and Michalakis 1999). The other parent is sampled with probability $1 / 2$, and the probability of identity between the two alleles is $Q_{\mathrm{fm}}$.

The second term in equation (12) accounts for the increase in local competition experienced by relatives of the focal juvenile and stems from this juvenile having a higher survival rate so that it is more likely to compete with other juveniles from its deme for open breeding spots. By having a higher survival probability and remaining philopatric, the focal juvenile may thus displace a fraction (1$m) / N_{u}$ of juveniles produced on the focal patch that are related to it by $\left(1+Q_{\mathrm{fm}}\right) / 4+Q_{\mathrm{fm}} / 2$, and it will displace a fraction $(1-m)\left(1-1 / N_{u}\right)$ of juveniles that descend from a different parent of sex $u$ that are related to it by $\left(Q_{u u}+Q_{\mathrm{fm}}\right) / 2$. The second term in equation (12) is thus the increase in kin competition due to increasing the gene expression of the allele donated by a parent of $\operatorname{sex} u$ in a focal juvenile.

Substituting the probabilities of genetic identity from "Probabilities of Identity by Descent" into the scaledrelatedness coefficients weighting the costs in equation (11) gives

$$
R_{b, \mathrm{f}}=R_{j, \mathrm{f}}
$$

and

$$
\begin{aligned}
R_{b, \mathrm{~m}}= & \frac{1}{2}\left\{\frac{1}{\tau} \frac{1+Q_{\mathrm{fm}}}{2}+\left(1-\frac{1}{\tau}\right)\left[\frac{1}{N_{\mathrm{m}}} \frac{1+Q_{\mathrm{fm}}}{2}\right.\right. \\
& \left.\left.+\left(1-\frac{1}{N_{\mathrm{m}}}\right) Q_{\mathrm{mm}}\right]+Q_{\mathrm{fm}}\right\} \\
- & \frac{(1-m)^{2}}{2}\left[\frac{1}{N_{\mathrm{m}}}\left(\frac{1+Q_{\mathrm{fm}}}{2}+Q_{\mathrm{fm}}\right)\right. \\
& \left.+\left(1-\frac{1}{N_{\mathrm{m}}}\right)\left(Q_{\mathrm{mm}}+Q_{\mathrm{fm}}\right)\right],
\end{aligned}
$$


where the only difference from equation (12) is that the inclusive fitness effects are functions of the probability of identity between a gene sampled in the focal juvenile derived from a male parent and a gene sampled randomly from a parent of the clutch of the juvenile (first line in eq. [14]), which is a decreasing function of the number of matings. Hence, $R_{b, \mathrm{~m}}<R_{b, \mathrm{f}}$ for $\tau>1$. Finally, we mention that $Q_{u v}$ simplifies so that

$$
Q_{u v}=\frac{(1-m)^{2}[N(r+\tau)-1]}{(1-m)^{2}[N(r+\tau)-1]+8 m(2-m) \tau r(1-r) N^{2}} .
$$

Because multiple paternity affects only the identity between a paternally derived allele in the focal offspring and an allele sampled randomly from one of the parents of its clutch, relatedness factors out of $\mathcal{S}_{x}$ and implies that $\mathcal{S}_{x}=0$ is equivalent to

$$
B_{\mathrm{f}}+B_{\mathrm{m}}-2 C=0 .
$$

Since the ES level of maternally derived gene expression $z_{a a}^{x}$ solves equation (16), $z_{a a}^{x}$ is independent of the demographic structure of the population and depends only on the balance of the costs and benefits of gene expression.

Setting $\mathcal{S}_{y}\left(z_{a a}^{y}\right)=0$ and using equation (8), we can show that $z_{a a}^{y}$ must satisfy

$$
\left(B_{\mathrm{f}}+B_{\mathrm{m}}\right)-2 C \times \frac{R_{b, \mathrm{~m}}}{R_{j s, \mathrm{~m}}}=0,
$$

where the ratio $R_{b, \mathrm{~m}} / R_{j s, \mathrm{~m}}$ varies between 0 (no indirect fertility cost to paternally derived gene expression) and 1 . When $\tau=1$ and there is no multiple paternity, paternally derived relatedness values for fertility and survival effects are also the same and $R_{j, \mathrm{~m}}=R_{b, \mathrm{~m}}$. Thus, $\mathcal{S}_{y}=\mathcal{S}_{x}$, which implies that $z_{a a}^{y}=z_{a a}^{x}$ and that selection is indifferent to Mendelian or imprinted gene expression.

When there is multiple paternity and $\tau>1$, the ratio $R_{b, \mathrm{~m}} / R_{j, \mathrm{~m}}$ decreases. Equation (17) shows that a smaller value of $R_{b, \mathrm{~m}}$ relative to $R_{i s, \mathrm{~m}}$ implies a reduction in the indirect fertility cost associated with paternally derived resource-enhancing gene expression, $\delta_{y}$, relative to the direct survival benefit. Although the strength of the reduction is a function of the demographic parameters of the model, such as the sex ratio, migration rate, and population size, a reduction of $R_{b, \mathrm{~m}} / R_{j s, \mathrm{~m}}$ always occurs as long as $\tau>1$. This results in $z_{a a}^{y}>z_{a a}^{x}$, because of the stronger selection pressure on paternally derived gene expression $\left(\mathcal{S}_{y}\right)$ than on maternally derived gene expression $\left(\mathcal{S}_{x}\right)$, and in silencing of the maternally derived allele.

\section{Sex-Specific Migration}

The results in the preceding section show that demographic parameters, including local deme size, migration rate, and sex ratio, have no effect on selection for imprinting of a resource-enhancing allele; multiple paternity is sufficient for exclusive expression by the paternally derived allele. The reason for this is that multiple paternity creates asymmetry between the relatedness of maternally and paternally derived alleles in the offspring of a given female. On average, maternally derived alleles are then more likely to be identical by descent within a clutch than are paternally derived ones (Haig 1992). Sex-specific dispersal may also generate asymmetry in relatedness and has been hypothesized to generate selection for imprinting (Haig 2000a). We study this proposition next.

In order to isolate the effect of sex-specific dispersal on relatedness from other factors, we assume that there is no multiple paternity $(\tau=1)$ and that there are an equal number of males and females within each deme $(r=$ $1 / 2$ and $\left.N_{\mathrm{f}}=N_{\mathrm{m}}=\mathcal{N}\right)$. The IBD probabilities $Q_{\mathrm{ff}}, Q_{\mathrm{ffm}}$, and $Q_{\mathrm{mm}}$ now take different values, since juveniles of different sexes migrate at different rates (see "Probabilities of Identity by Descent"). Under these conditions, the selection gradients on the mutant allele resulting from its expression when paternally and maternally inherited are given, respectively, by

$$
\begin{aligned}
& S_{x}=\frac{1}{2}\left[\left(B_{\mathrm{f}}-C\right) R_{j s, \mathrm{ff}}+\left(B_{\mathrm{m}}-C\right) R_{j s, \mathrm{mf}}\right], \\
& S_{y}=\frac{1}{2}\left[\left(B_{\mathrm{f}}-C\right) R_{j s, \mathrm{fm}}+\left(B_{\mathrm{m}}-C\right) R_{j s, \mathrm{~mm}}\right],
\end{aligned}
$$

where

$$
\begin{aligned}
R_{j s, u v}= & \frac{1}{2}\left(\frac{1+Q_{\mathrm{fm}}}{2}+Q_{\mathrm{fm}}\right)-\frac{\left(1-m_{u}\right)^{2}}{2} \\
& \times\left[\frac{1}{\mathcal{N}}\left(\frac{1+Q_{\mathrm{fm}}}{2}+Q_{\mathrm{fm}}\right)+\left(1-\frac{1}{\mathcal{N}}\right)\left(Q_{v v}+Q_{\mathrm{fm}}\right)\right]
\end{aligned}
$$

is a scaled imprinted measure of relatedness that depends on sex-specific migration probabilities.

The interpretation of this relatedness coefficient is similar to that of equation (12): the first term in parentheses is the probability of identity between an allele sampled in a focal offspring of sex $u$, which was inherited from a parent of $\operatorname{sex} v$, and an allele sampled in a random parent of that offspring. The term in square brackets in equation (19) is the probability of identity between an allele sampled 
in the focal offspring and an allele sampled from a random parent that has produced an offspring entering in competition with the focal offspring, which occurs with probability $\left(1-m_{u}\right)^{2}$. The second term in equation (19) thus reflects the loss in inclusive fitness by an individual carrying the mutant allele stemming from how the mutant increases the intensity of local competition.

The first thing to note about equation (18) is that sexspecific selection, $B_{\mathrm{f}} \neq B_{\mathrm{m}}$, and migration, $m_{\mathrm{f}} \neq m_{\mathrm{m}}$, are necessary for there to be selection for imprinting. If there is no sex-specific selection $\left(B_{\mathrm{f}}=B_{\mathrm{m}}=B\right)$, then $\mathcal{S}_{x}$ and $\mathcal{S}_{y}$ are both proportional to $B-C$ and $z_{a a}^{x}=z_{a a}^{y}$. Although sex-specific migration may generate asymmetry in relatedness between maternally and paternally derived alleles, these differences do not affect the trade-off between the fertility costs and the survival benefits when survival is independent of sex. When migration is independent of sex, $m_{\mathrm{f}}=m_{\mathrm{m}}=m$, relatedness among surviving adults is independent of sex, $R_{j s, \mathrm{ff}}=R_{j s, \mathrm{mf}}=R_{j s, \mathrm{fm}}=R_{j s, \mathrm{~mm}}$, and the benefits and costs of gene expression to survival and fertility are the same for both maternally and paternally derived alleles. This means that $\mathcal{S}_{x}=\mathcal{S}_{y}$ and, again, $z_{a a}^{x}=z_{a a}^{y}$. In order to generate selection for imprinting, sex-specific viability and migration must co-occur so that the trade-off between the benefits and costs of gene expression is different between maternally and paternally derived alleles.

Recall that the maternally derived allele is silenced when $z_{a a}^{y}-z_{a a}^{x}>0$ and the paternally derived allele is silenced when $z_{a a}^{y}-z_{a a}^{x}<0$ (see "Direction of Selection on Imprinting and Evolutionary Stability"). The effect of sexspecific migration on the direction on imprinting can be discerned by determining which of the selection gradients, $\mathcal{S}_{x}\left(z_{a a}\right)$ or $\mathcal{S}_{y}\left(z_{a a}\right)$, vanishes first as the level of gene expression $z_{a a}$ increases. We perform this analysis in "SexSpecific Migration and Change in Frequency of $A$ " in the online edition of the American Naturalist and show that the strength of sex-specific selection, independent of which sex migrates more often, determines which of $z_{a a}^{x}$ or $z_{a a}^{y}$ is larger. That is, the maternally derived allele is silenced if

$$
B_{\mathrm{f}}-B_{\mathrm{m}}>0
$$

and if the reverse inequality holds, the paternally derived allele is silenced.

That the sex bias in viability selection, and not migration, determines which allele is silenced is due to the symmetric effects of migration on the local-competition components of the $R_{j s, w}$ coefficients defined in equation (19). If one sex migrates less frequently than the opposite sex (i.e., the first sex is philopatric), this increases the chance that both juveniles and adults of the philopatric sex will compete with relatives locally, which decreases relatedness coefficients that measure identity between alleles of the philopatric sex. Since we assume an even sex ratio, the magnitude of this increase in local competition is the same for females when migration has a female bias of a certain amount as it is for males when migration has a male bias of the same amount. This means that sex biases in migration affect the selection gradients in equation (18) symmetrically, leaving the sex bias in viability selection to determine which allele is silenced and which is expressed.

More generally for $\tau \geq 1$ and any sex ratio, which allele is silenced by a particular sex bias in viability selection is determined by whether the effect of local competition on relatedness is stronger when philopatric alleles are found in both parent and offspring or are found apart. If the increase in local competition for alleles donated by parents of the philopatric sex to offspring of that sex, which can be measured by the geometric mean $\left(R_{j s, \mathrm{ff}} R_{j s, \mathrm{~mm}}\right)^{1 / 2}$, is weaker than that for alleles where only one of the donating parent or the offspring is of the philopatric sex, measured by $\left(R_{j s, \mathrm{mf}} R_{j s, \mathrm{fm}}\right)^{1 / 2}$, then $R_{j s, \mathrm{ff}} R_{j s, \mathrm{~mm}}>R_{j s, \mathrm{mf}} R_{j s, \mathrm{fm}}$. In this case, stronger viability selection on males, $B_{\mathrm{m}}>B_{\mathrm{f}}$, leads to expression of paternally derived alleles and silencing of maternally derived alleles, and the opposite expression pattern is expected for stronger viability selection on females, $B_{\mathrm{f}}>B_{\mathrm{m}}$. The effect of sex-specific viability is reversed when the increase in local competition is weaker for alleles for which only one of the donating parent or the offspring is of the philopatric sex; that is, $R_{j s, \mathrm{ff}} R_{j s, \mathrm{~mm}}<R_{j s, \mathrm{mf}} R_{j s, \mathrm{fm}}$ means that $B_{\mathrm{m}}>B_{\mathrm{f}}$ implies silencing of paternally derived alleles, and $B_{\mathrm{f}}>B_{\mathrm{m}}$ means silencing of maternally derived alleles. The latter case leads to inequality (20) when $\tau=1$ and $r=1 / 2$.

By itself, an uneven sex ratio strongly affects the degree of population structure (this can be seen in fig. A1, in the online edition of the American Naturalist); for this case, $r \neq 1 / 2$, sex-specific migration does play a role in determining which allele is imprinted. Since the expression for $\Delta p$ is sufficiently complex in this case, we present numerical results in figure 2 that show which gene expression level, $z_{a a}^{x}$ or $z_{a a}^{y}$, is greater under a range of sex ratios and sex-biased migration rates. We assume that $N=10$ and $m=0.01$ for the nonphilopatric sex and that viability selection affects males more strongly than females, $B_{\mathrm{m}}>B_{\mathrm{f}}$. Focusing on the $\tau=1$ line, we can see that, as expected, $z_{a a}^{x}>z_{a a}^{y}$ for all migration rates when $r=1 / 2$. If the sex ratio is female biased $(r<1 / 2)$, then $z_{a a}^{x}>z_{a a}^{y}$ for all levels of female-biased migration, which we see in the left-hand plot. If migration is male biased, then $z_{a a}^{y}>z_{a a}^{x}$ when this bias is weak and $z_{a a}^{x}>z_{a a}^{y}$ when this bias is strong. The level of male bias in migration required for $z_{a a}^{x}$ to become greater than $z_{a a}^{y}$ increases as the sex ratio become more female biased to the point where $z_{a a}^{y}>z_{a a}^{x}$ for any level of male-biased migration. For male-biased sex ratios, the pat- 

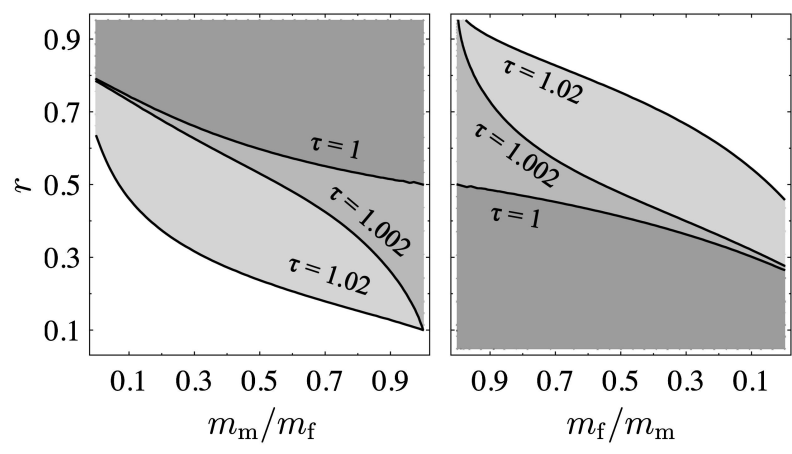

Figure 2: Sign of $z_{a a}^{y}-z_{a a}^{x}$ for $h=1 / 2$ and $N=10$ as a function of sexspecific migration and the sex ratio when there is no generation overlap. In the left-hand plot, $m_{\mathrm{f}}=0.01$, and in the right-hand plot, $m_{\mathrm{m}}=$ 0.01 . We assume that $B_{\mathrm{m}}>B_{\mathrm{f}}$. In the left-hand plot, the region above a given $\tau$ contour line indicates $z_{a a}^{y}-z_{a a}^{x}>0$, and the region below the contour line indicates $z_{a a}^{y}-z_{a a}^{x}<0$. The pattern is reversed in the righthand plot, where the region below a given $\tau$ contour line indicates $z_{a a}^{y}-z_{a a}^{x}>0$, and the region above the contour line indicates $z_{a a}^{y}-z_{a a}^{x}<$ 0 .

tern is reversed: any male-biased migration yields $z_{a a}^{x}>$ $z_{a a}^{y}$, and female-biased migration yields $z_{a a}^{x}>z_{a a}^{y}$ only when the migration bias is sufficiently strong. Figure 3 shows that this qualitative pattern holds across a range of populations sizes and migration rates. An analogous pattern also results when viability selection affects females more strongly and $z_{a a}^{y}>z_{a a}^{x}$ is predicted at an even sex ratio. Thus, condition (20) holds when the sex ratio and migration rates are biased toward the same sex or when migration is strongly biased and the sex ratio is close to even.

Figure 2 also shows what happens when $\tau$ increases beyond 1 and females mate multiply. When migration is either weakly male or female biased, multiple paternity immediately results in the classical result of silencing the maternally derived allele for almost all values of $r$. As the sex ratio and migration rates become increasingly biased toward the same sex, $z_{a a}^{x}>z_{a a}^{y}$ and the paternally derived allele is silenced. As the degree of multiple paternity increases, the threshold levels of bias in the sex ratio and migration rates required for this shift rapidly increase; long before $\tau=2$, no amount of bias in the sex ratio and migration rates can generate silencing of the paternally derived allele. In addition, figure 3 shows that increasing the population size makes it much more difficult to reverse the classical result and generate lower ES gene expression from the paternally derived allele. Taken together, these results reveal that sex-biased migration rates and sex ratios can counterbalance the effect of a small degree of multiple paternity on patrilineal relatedness when viability selection is stronger on males and the population size is small.

\section{Overlapping Generations}

Asymmetry in relatedness between males and females can be produced by differential adult survival when generations are overlapping. If adult individuals of one sex have a lower mortality than adults of the other sex, then alleles drawn from individuals of the first sex are more likely to be identical by descent than alleles drawn from the other sex. If this effect on relatedness is strong enough, it may generate selection for imprinted gene expression and possibly result in silencing of the paternally derived allele even when there is multiple paternity.

Assuming that males and females disperse at equal rates and that there is no multiple paternity $(\tau=1)$, the relatedness coefficients in the selection gradients in equations (7) and (8) simplify so that $R_{j s, \mathrm{fu}}=R_{\mathrm{js}, \mathrm{m} u}=R_{j s, u}$ $R_{b, f u}=R_{b, \mathrm{~m} u}=R_{b, u}$ and $R_{b, u}=R_{j s, u}$ which is given by equation (12). Therefore,

$$
\begin{gathered}
\mathcal{S}_{x}=\alpha_{\mathrm{f}} s_{\mathrm{f}} B_{\mathrm{f}} R_{a s, \mathrm{ff}}+\alpha_{\mathrm{m}} s_{\mathrm{m}} B_{\mathrm{m}} R_{a s, \mathrm{mf}} \\
+\beta\left(B_{\mathrm{f}}+B_{\mathrm{m}}-C\right) R_{\mathrm{js}, \mathrm{f}}, \\
\mathcal{S}_{y}=\alpha_{\mathrm{f}} \mathcal{s}_{\mathrm{f}} B_{\mathrm{f}} R_{a s, \mathrm{fm}}+\alpha_{\mathrm{m}} s_{\mathrm{m}} B_{\mathrm{m}} R_{a s, \mathrm{~mm}} \\
+\beta\left(B_{\mathrm{f}}+B_{\mathrm{m}}-C\right) R_{j s, \mathrm{~m}} .
\end{gathered}
$$

We show in "Selection Gradients and Relatedness" in the online edition of the American Naturalist and "Probabilities of Identity by Descent" that the remaining coefficient in these selection gradients, $R_{a s, u v}$, which is due to the survival of adults from one generation to the next, is given by

$$
\begin{aligned}
& R_{a s, u v}= \frac{1+Q_{\mathrm{fm}}}{2}-\frac{(1-m)}{2} \\
& \times\left[\frac{1}{N_{u}}\left(\frac{1+Q_{\mathrm{fm}}}{2}+\frac{Q_{\mathrm{f}[v] \mathrm{m}[v]}+Q_{u[v] \tilde{u}[v]}}{2}\right)\right. \\
&+\left(1-\frac{1}{N_{u}}\right)\left(\frac{Q_{u[f] u[v]}+Q_{u[v] u[\mathrm{~m}]}}{2}\right. \\
&\left.\left.+\frac{Q_{\mathrm{f}[v] \mathrm{m}[v]}+Q_{u[v] \tilde{u}[\tilde{v}]}}{2}\right)\right],
\end{aligned}
$$

where $Q_{u[\tilde{u} v v[\tilde{v}]}$ are imprinted IBD coefficients that measure the probability that an allele in an adult of sex $u$ that came from a parent of sex $\tilde{u}$ is identical to an allele from another adult of sex $v$ that came from a parent of sex $\tilde{v}$. Methods for calculating such imprinted IBD coefficients are given in "Probabilities of Identity by Descent."

As was the case above, the scaled relatedness given by 
$N=10$
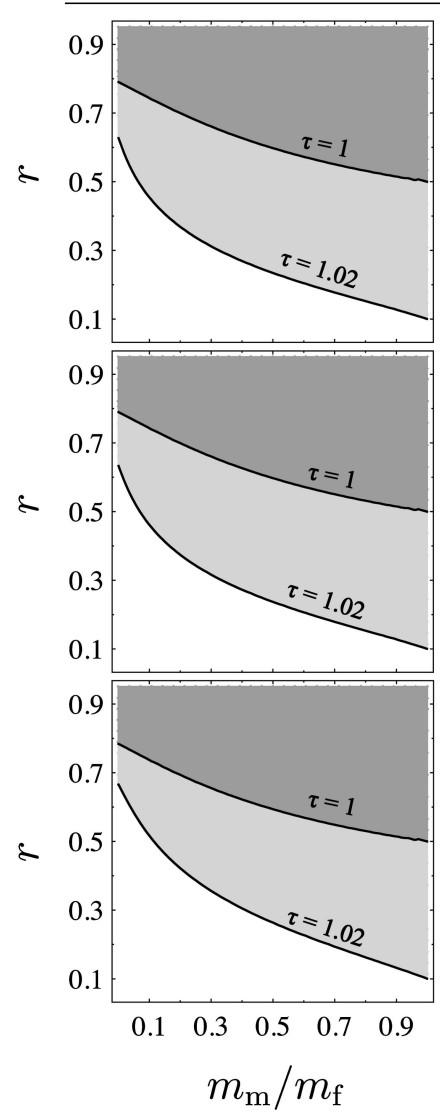
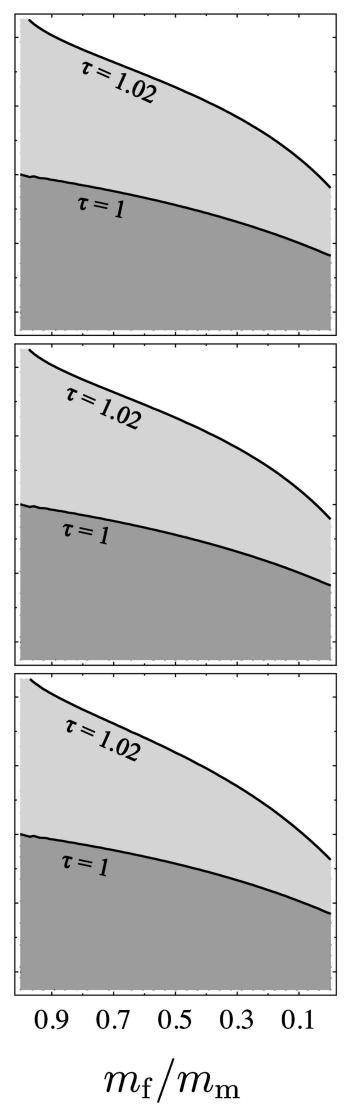

$N=100$

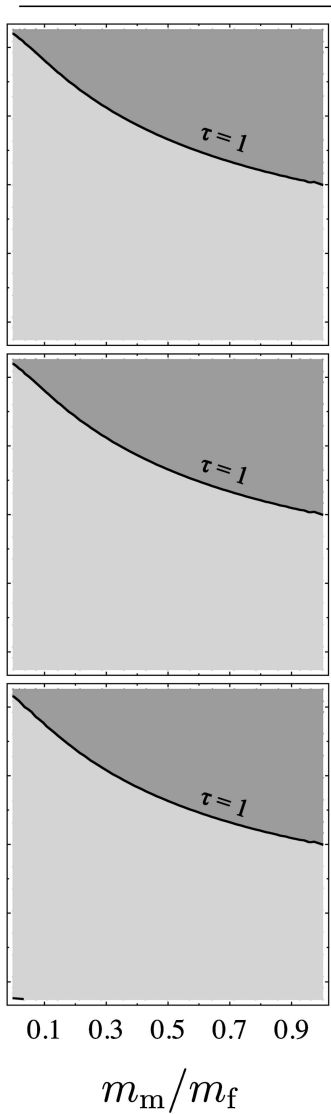

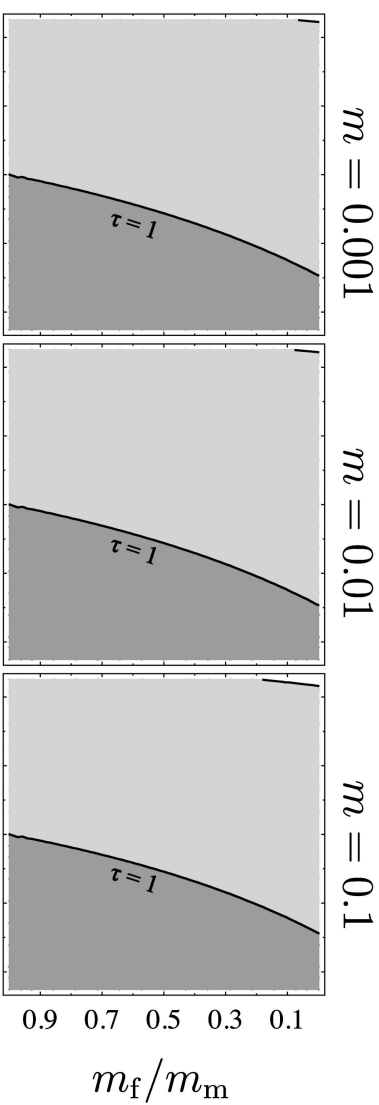

Figure 3: Same as figure 2, except that we vary the magnitude of the migration rate and the population size. In first and third columns, $m_{\mathrm{f}}=m$, where $m$ is given on the right-hand side of the plots, and in second and fourth columns, $m_{\mathrm{m}}=m$. In the first and third columns, the region above a given $\tau$ contour line indicates $z_{a a}^{y}-z_{a a}^{x}>0$, and the region below the contour line indicates $z_{a a}^{y}-z_{a a}^{x}<0$. The pattern is reversed in the second and fourth columns, where the region below a given $\tau$ contour line indicates $z_{a a}^{y}-z_{a a}^{x}>0$ and the region above the contour line indicates $z_{a a}^{y}-z_{a a}^{x}<0$. The population size is $N=10$ for the first two columns and $N=100$ for the last two columns.

equation (22) accounts for two different types of inclusive fitness effects accruing to an individual carrying the mutant allele. The first term, $\left(1+Q_{\mathrm{fm}}\right) / 2$, is the probability of identity between an allele sampled in an adult of sex $u$ (the actor), which was inherited from a parent of $\operatorname{sex} v$, and the adult itself (the adult is here also the recipient of the act). The second term in equation (22) accounts for the increase in local competition affecting the relatives of the actor when it has a higher adult survival probability through an increase in gene expression level. Then, all offspring produced in the focal patch that remain philopatric (a fraction $1-m$ of the productivity of the focal patch, which includes the offspring of the focal actor) are less likely to find an open breeding space. The increase in survival of the focal adult then displaces offspring, which are related to it by the relatedness term given in square brackets in equation (22) divided by $1 / 2$.
Given the selection gradients in equation (21), we show in "Overlapping Generations" in the online edition of the American Naturalist that the maternally derived allele is silenced if

$$
\frac{\alpha_{\mathrm{f}} s_{\mathrm{f}} R_{a s, \mathrm{fm}}+\alpha_{\mathrm{m}} s_{\mathrm{m}} R_{a s, \mathrm{~mm}}}{R_{j s, \mathrm{~m}}}-\frac{\alpha_{\mathrm{f}} s_{\mathrm{f}} R_{a s, \mathrm{ff}}+\alpha_{\mathrm{m}} s_{\mathrm{m}} R_{a s, \mathrm{mf}}}{R_{j s, \mathrm{f}}}>0,
$$

and if the reverse inequality holds, the paternally derived allele is silenced. The numerator of the first term in inequality (23) measures the relatedness between a paternally derived allele in a random surviving adult and a random allele in that adult where surviving males and females are sampled according to their reproductive value-weighted survival probabilities. The numerator of the second term 
is interpreted analogously for maternally derived alleles. The denominators are the relatedness values between paternally and maternally derived alleles in offspring and random parents. Thus, inequality (23) says that evolutionarily stable gene expression of the paternally derived allele will be larger than expression of the maternally derived allele when paternally derived alleles have a higher relatedness in surviving adults relative to random adults than do maternally derived alleles. In effect, higher patrilineal relatedness among surviving adults selects for higher expression of the paternally derived allele, since survival rates increase with gene expression levels.

To evaluate the effect of the demographic parameters on the relatedness values in inequality (23), we resort to numerical analysis, since the IBD equations are quite complicated. For a range of female and male survival probabilities, figure 4 shows which of $z_{a a}^{x}$ or $z_{a a}^{y}$ is greater for $N=10$ and $m=0.01$. The sex ratio is female biased in the left-hand plot, male biased in the right-hand plot, and even in the middle plot. Above a given $\tau$ contour line, $z_{a a}^{x}>z_{a a}^{y}$, and $z_{a a}^{y}>z_{a a}^{x}$ below the contour line. Focusing on the even-sex-ratio case in the middle plot and the $\tau=$ 1 (no multiple paternity) line, we can see that for weak generation overlap (small $s_{\mathrm{f}}$ and $s_{\mathrm{m}}$ ) with any sex bias, $z_{a a}^{x}>z_{a a}^{y}$, which results in silencing of the paternally derived allele. This effect is likely due to the fact that when females mate only once, the probability that two random offspring share a mother, $1 / N_{\mathrm{f}}$, is lower than the probability of sharing a father, $1 / N_{\mathrm{f}}+1 / N_{\mathrm{m}}\left(1-1 / N_{\mathrm{f}}\right)$, which increases kin competition among patrilineal lines. This increase in kin competition decreases $R_{a s, \mathrm{fm}}$ and $R_{a s, \mathrm{~mm}}$ relative to $R_{a s, \mathrm{ff}}$ and $R_{a s, \mathrm{mf}}$ for small amounts of generation overlap. High survival rates in females and low rates in males can counterbalance this effect by strengthening kin competition among matrilineal lines enough to yield $z_{a a}^{y}>z_{a a}^{x}$.
For both female- and male-biased sex ratios, high female and low male survival yield $z_{a a}^{y}>z_{a a}^{x}$ and maternal silencing. Even so, the direction of sex ratio bias changes the degree of bias in survival rates needed for $z_{a a}^{y}>z_{a a}^{x}$. A female-biased sex ratio requires a smaller female bias in survival rates in order for $z_{a a}^{y}>z_{a a}^{x}$ than does a male-biased sex ratio. At first, this seems surprising, since a femalebiased sex ratio should increase the effect of kin competition among patrilineal lines, which would further decrease $R_{a s, \mathrm{fm}}$ and $R_{a s, \mathrm{~mm}}$ relative to $R_{a s, \mathrm{ff}}$ and $R_{a s, \mathrm{mf}}$. However, the effect of the sex ratio on kin competition is stronger on the within-generation relatedness values $R_{j \mathrm{~s}, \mathrm{~m}}$ and $R_{j s, \mathrm{f}}$, where $R_{j s, \mathrm{~m}}$ decreases relative to $R_{j \mathrm{~s}, \mathrm{f}}$ for female-biased sex ratios. Thus, a female-biased sex ratio increases patrilineal relatedness among surviving adults relative to random adults, which increases the scope for silencing of the maternal allele. For analogous reasons, a male-biased sex ratio makes it easier to silence the paternal allele.

Figure 4 also shows what happens to the ES expression levels when multiple paternity is rare. Regardless of the sex-ratio bias, multiple paternity reduces the bias in survival rates needed for maternal silencing. Figure 5 suggests that, as was the case for sex-specific migration, the strength of the effect of multiple paternity is roughly independent of the migration rate but increases with increasing population size. Increasing $\tau$ to 2 (not shown) under the sex ratios used in figure 4 erases any opportunity for $z_{a a}^{x}>$ $z_{a a}^{y}$, even for $N=10$. Taken together, these results suggest that for mating systems that are close to monogamous, the reverse pattern of the classical result, paternal silencing of a resource-enhancing gene, should hold in small or moderate-sized populations as long as the survival rates and the sex ratio are not too female biased. When there is a significant degree of multiple paternity, we predict

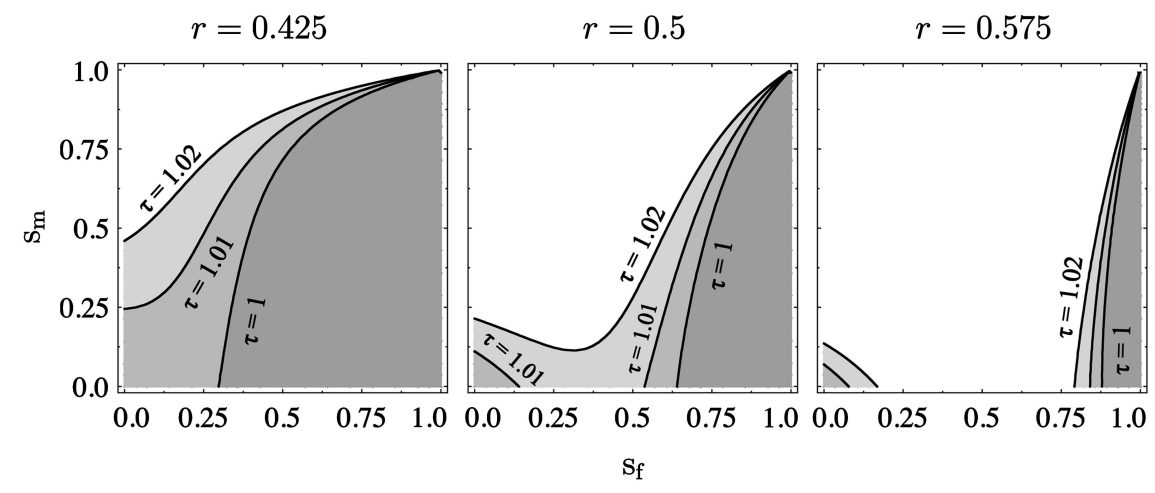

Figure 4: Sign of $z_{a a}^{y}-z_{a a}^{x}$ for $h=1 / 2, N=10$, and $m_{\mathrm{f}}=m_{\mathrm{m}}=0.01$ as a function of female and male adult survival rates, $s_{\mathrm{f}}$ and $s_{\mathrm{m}}$, respectively. The value of the sex ratio is listed at the top of each plot. The region below a given $\tau$ contour line indicates $z_{a a}^{y}-z_{a a}^{x}>0$, and the region above the contour line indicates $z_{a a}^{y}-z_{a a}^{x}<0$. 

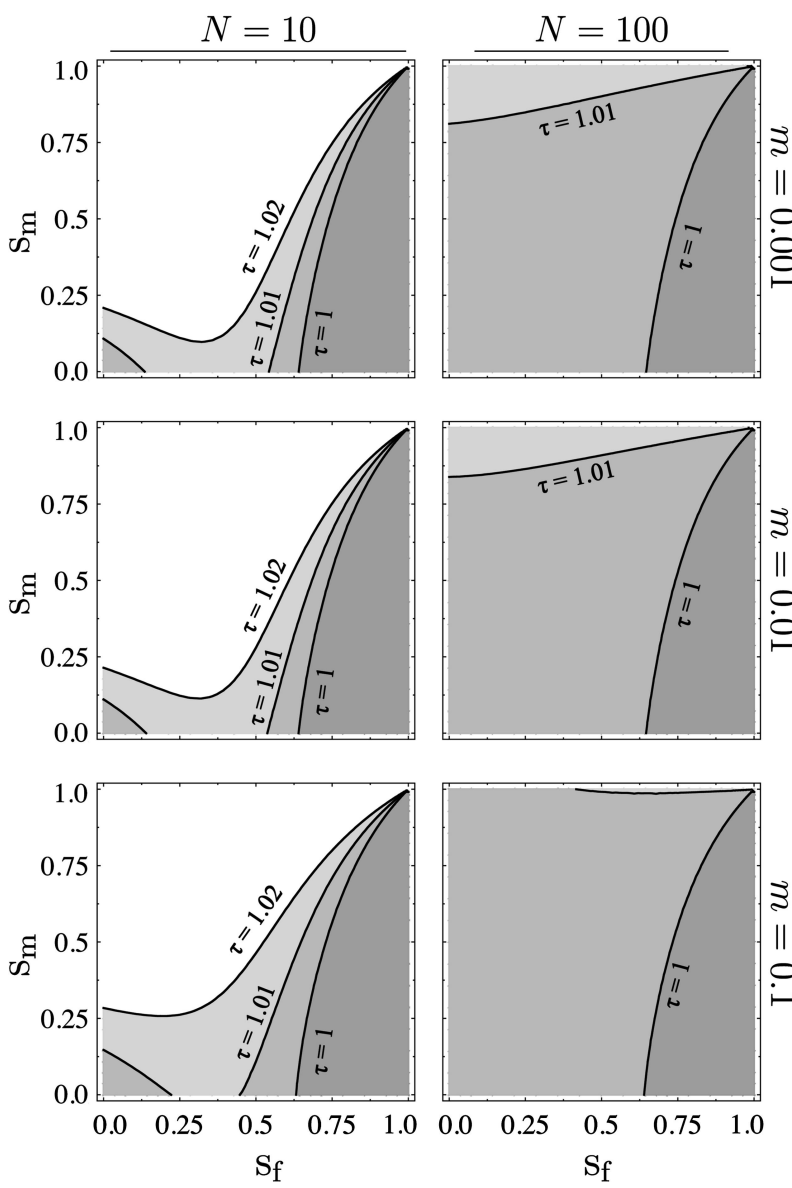

Figure 5: Same as figure 4, except that we vary the magnitude of the migration rate and the population size, and the sex ratio is $r=0.5$. The population sizes are listed above the columns and the migration rates on the right, where $m_{\mathrm{f}}=m_{\mathrm{m}}=m$. The region below a given $\tau$ contour line indicates $z_{a a}^{y}-z_{a a}^{x}>0$, and the region above the contour line indicates $z_{a a}^{y}-z_{a a}^{x}<0$.

maternal silencing independent of the other demographic parameters.

\section{Discussion}

The original formulation of the kinship hypothesis for the evolution of genomic imprinting used inclusive fitness theory to show that the asymmetry in relatedness between maternally and paternally derived alleles generated by multiple paternity can lead to silencing of genes that enhance offspring growth if this growth benefits offspring individually but comes at the cost of future female fertility (Haig 1992, 1997). Later models showed that the same result holds when offspring growth decreases current female fertility because of scramble competition (Mochizuki et al. 1996; Haig and Wilkins 2000; Kondoh and Higashi 2000;
Greenwood-Lee et al. 2002). All of these models, however, assumed a family-structured population and additive interactions between alleles. They did not address how genomic imprinting might evolve as a result of relatedness asymmetries induced by local genetic drift due to finite population size and migration, by dominance, by sexspecific effects on migration and selection, or by generation overlap. The model presented in this article addresses these issues by embedding the basic life-history assumption of the kinship hypothesis within an island model of population structure.

\section{Multiple Paternity}

If there is no generation overlap and migration is independent of sex, our model reproduces the predictions of the classic kinship hypothesis: multiple paternity results in silencing of the maternally derived copy of the resourceenhancing gene and full expression of the paternally derived copy. This analysis easily extends to resource-inhibiting genes and predicts that such loci will be expressed by the maternally derived copy if they have opposite effects on survival and fertility to resource-enhancing genes. These results are independent of the demographic parameters of the model, including local deme size, migration rate, and sex ratio; since these parameters determine the degree of population structure due to local drift, we can conclude that local drift within a deme does not affect long-term predictions of which allele will be silenced. The reason that the degree of multiple paternity has such a strong effect even in small local populations is that any amount of asymmetry between the matrilineal and patrilineal relatednesses of developing offspring of a given female will result in asymmetric fertility costs of gene expression from maternally and paternally derived alleles; such costs result in different "optimal" levels of expression from maternally and paternally derived alleles and in a convergence-stable level of gene expression at which one allele is silenced (see fig. 1).

It is important to note, however, that local drift and population structure will have an effect on the strength of selection for silencing of maternally derived alleles. For a given level of multiple paternity, the effect of multiple paternity decreases rapidly with small deme size, a femalebiased sex ratio, and weak migration, all of which increase local drift and decrease the efficacy of selection. Selection can also be weak when the fertility costs and survival benefits of gene expression are weak. If selection is weak, it may take a long time for imprinted gene expression to evolve, since advantageous mutants will reach fixation at a rate only slightly higher than that of neutral mutants. This could allow for transient heterogeneity in imprinting status within a population, as has been observed in some 
genes (Ogawa et al. 1993). It is possible that such heterogeneity could also be due to selection for a polymorphism, although we have not explored the possibility of such polymorphisms in our model because of overdominance in gene expression.

\section{Sex-Specific Migration}

As with multiple paternity, sex-specific migration can generate relatedness asymmetries among alleles sampled from different sexes. Haig (2000a) suggested that sex-specific migration can generate selection for imprinting due to such asymmetries. After including sex-specific migration in our model, we find that when females are monogamous and alleles interact additively, sex-specific migration is not sufficient to generate selection for imprinting. Once migration occurs at different rates in the sexes, sex-specific selection is required to generate selection for imprinting, and the direction of sex-specific selection, not migration, is a better predictor of which allele is silenced. Specifically, when there is an even sex ratio, the sex experiencing stronger viability selection silences the resource-enhancing allele that it donates to offspring.

Sex-specific selection drives selection for imprinted gene expression because of the effect of sex-specific migration on competition within the sexes for resources or breeding sites during the density-dependent regulation phase of the life cycle. All else being equal, sex biases in migration affect the strength of local competition on each sex symmetrically, in the sense that a given level of philopatry in males increases local competition among alleles in males as much as the same level of philopatry in females increase local competition among alleles in females. Since the selection gradients on maternally and paternally derived alleles both depend on relatedness between alleles in males and females, the sex bias in selection determines which allele is silenced. When selection is stronger on one sex, this results in an increased benefit to gene expression of an allele donated by a parent of the second sex and silencing of the expression of the allele donated by a parent of the first sex, regardless of which sex is philopatric.

\section{Sex-Specific Selection}

Even in panmictic populations, sex-specific selection can generate selection for imprinting. In a two-locus population genetic model, Day and Bonduriansky (2004) found that a modifier allele that causes silencing of maternally derived alleles increases in frequency when selection is stronger on males. Van Cleve and Feldman (2007) confirmed this result as a special case when there is no dominance at the locus under selection. In these models, imprinting can be seen as a way for phenotypes to more closely track selective pressures; the sex experiencing stronger selection will pass on to its offspring a higher frequency of the allele better adapted in that sex. In a quantitative genetic model of the evolution of X-linked genomic imprinting, Iwasa and Pomiankowski (2001) found a socalled reversed pattern, in which stronger selection on one sex resulted in silencing of the alleles that that sex had donated to offspring. Their model contained the same lifehistory trade-off between female fertility and offspring survival due to increased offspring gene expression found in the classic kinship hypothesis and our model. The reversed pattern in X-linked imprinting was due to the fact that males obtain their only X chromosome from their mothers. If viability selection is stronger on males than on females, then expression of maternally derived alleles will be higher than expression from paternally derived ones (and vice versa). Our model contains the same reversed pattern, except that it derives from the effects of kin competition and sex-specific migration. In principle, it is possible to reconcile these two patterns by including in our model a modifier locus that controls the imprinting status of a major locus whose expression level has different effects on survival and fertility. It is likely that in this case, recombination between the modifier and major loci will be crucial in determining whether the normal or reversed pattern of imprinting evolves.

\section{Generation Overlap}

Our model shows how generation overlap affects patrilineal and matrilineal relatedness and how asymmetries in these relatedness values can drive silencing of either the paternally derived or the maternally derived resourceenhancing allele. Specifically, we show how a monogamous mating system can increase local competition among patrilines, which yields silencing of the paternally derived allele for small degrees of generation overlap. This is in contrast to the classical result, which predicts that any pattern of gene expression, for example, silencing of either allele or equal expression from both, is ES under monogamy (Haig and Wilkins 2000). The amount of generation overlap due to sex-specific survival of adults also affects local competition in demes. Male bias in adult survival rates generates more local competition among patrilines and results in silencing of the paternally derived allele. Likewise, female bias in adult survival rates can increase local competition among matrilines enough to counter the effect of monogamy on patrilines and result in maternal silencing. Female-biased sex ratios should yield more intense local competition among patrilines, but surprisingly, female-biased sex ratios make paternal silencing harder. This is due to the fact that sex ratio bias has a stronger local competitive effect on fertility than on adult survival. 
Our results suggest that when multiple paternity is infrequent, paternal silencing of a resource-enhancing gene can result as long as the sex ratio and adult survival rates are not too female biased.

\section{Conclusions}

In studying the kinship hypothesis in a subdivided population, we find that the trade-off between female fertility and offspring survival and significant levels of multiple paternity are sufficient to generate selection for imprinting. This occurs despite the effects that other demographic variables, including local deme size, migration, and sex ratio, have on population structure. When multiple paternity is infrequent, we find that the demographic variables become crucial in determining which allele will be expressed and which silent in the evolutionary long term. In fact, the sex ratio, sex-specific selection, migration, and adult survival can all affect which allele is imprinted. Moreover, our results suggest that many perturbations in the sex symmetry of demographic variables will lead to genomic imprinting. Support for this idea comes from recent work by Úbeda and Gardner (forthcoming) that predicts that genes for helping behaviors will be imprinted in a manner dependent on sex biases in migration or other demographic factors. If sex biases in demography can often generate selection for imprinting, then a reasonable next step in evaluating genomic imprinting in an evolutionary context is to determine why so few genes are imprinted (Franklin et al. 1996; Spencer 2000). It is possible that the recessivity of most deleterious mutations could partly explain this (Haig 2000b; Van Cleve and Feldman 2007), but more genomic work is needed to confirm this conjecture.

Some of the strongest empirical support for the kinship hypothesis comes from the fact that, among vertebrates, eutherians (placental mammals) and marsupials but not monotremes (platypus and echidna) are known to harbor imprinted genes (Ferguson-Smith and Surani 2001). Among the known mammalian imprinted genes, many have effects related to growth or morphogenesis (Morison et al. 2005) and are active in placental tissue (Coan et al. 2005). However, a systematic test of the kinship hypothesis will require genomewide data on gene function and imprinting status across a range of mammalian species with diverse mating systems and life histories. Genomewide data on imprinting status using high-throughput sequencing technologies have been collected for mice (Wang et al. 2008) but remain to be collected for nonmodel mammal species. Once such data are available, demographically explicit models like the one we present here will become essential for testing quantitative predictions about patterns in imprinting status across the mammalian phylogeny.

Such demographically explicit models will allow us to use fixation probabilities and data on generation times to estimate how a variety of demographic and life-history variables affect the timescales required for the evolution of imprinting. In addition, we will be able to quantitatively assess the relative importance of different demographic factors in driving the evolution of imprinting in different species. For example, our model suggests that when multiple paternity is uncommon, we should be able to predict imprinting status on the basis of other demographic variables. Although the degree of multiple paternity required for these variables to be important in our model is small, this is due to our assumption that competition for maternal resources is strong; in the likely case that competition for maternal resources is weaker (and better modeled by contest competition instead of scramble competition: see Mochizuki et al. 1996; Haig and Wilkins 2000; Kondoh and Higashi 2000; Greenwood-Lee et al. 2002), demographic variables will be more important in predicting imprinting status. If genomewide searches for imprinted genes find them in nonmammalian species such as birds, demographic factors will likely become an important component of explaining imprinting in these species, since conflict over maternal resources is weaker.

Generally, basic demography and life-history features, such as generation overlap and sex bias in dispersal, are crucial to a more detailed and broader view of the phenotype of interest. Our model demonstrates this to be the case for genomic imprinting and suggests that such a detailed approach will be fruitful in the study of related problems, including parent-offspring conflict and parental care.

\section{Acknowledgments}

J.V. was supported by National Library of Medicine Training Grant LM-07033. This research was supported in part by National Institutes of Health grant GM-28016 to M.W.F.

\section{Literature Cited}

Bartolomei, M. S., and S. M. Tilghman. 1997. Genomic imprinting in mammals. Annual Review of Genetics 31:493-525.

Charnov, E. L. 1982. The theory of sex allocation. Monographs in Population Biology 18. Princeton University Press, Princeton, NJ.

Coan, P. M., G. J. Burton, and A. C. Ferguson-Smith. 2005. Imprinted genes in the placenta: a review. Placenta 26(suppl. A):S10-S20.

Day, T., and R. Bonduriansky. 2004. Intralocus sexual conflict can drive the evolution of genomic imprinting. Genetics 167:15371546.

Eshel, I. 1983. Evolutionary and continuous stability. Journal of Theoretical Biology 103:99-112.

Ferguson-Smith, A. C., and M. A. Surani. 2001. Imprinting and the epigenetic asymmetry between parental genomes. Science 293: 1086-1089. 
Frank, S. A. 1998. Foundations of social evolution. Princeton University Press, Princeton, NJ.

Franklin, G. C., G. I. Adam, and R. Ohlsson. 1996. Genomic imprinting and mammalian development. Placenta 17:3-14.

Gandon, S., and Y. Michalakis. 1999. Evolutionarily stable dispersal rate in a metapopulation with extinctions and kin competition. Journal of Theoretical Biology 199:275-290.

Greenwood-Lee, J., P. Taylor, and D. Haig. 2002. The inclusive fitness dynamics of genomic imprinting. Selection 2:103-118.

Haig, D. 1992. Genomic imprinting and the theory of parentoffspring conflict. Seminars in Developmental Biology 3:153-160.

- 1997. Parental antagonism, relatedness asymmetries, and genomic imprinting. Proceedings of the Royal Society B: Biological Sciences 264:1657-1662.

- 2000a. Genomic imprinting, sex-biased dispersal, and social behavior. Annals of the New York Academy of Sciences 907:149163.

- 2000b. The kinship theory of genomic imprinting. Annual Review of Ecology and Systematics 31:9-32.

Haig, D., and J. F. Wilkins. 2000. Genomic imprinting, sibling solidarity and the logic of collective action. Philosophical Transactions of the Royal Society B: Biological Sciences 355:1593-1597.

Hamilton, W. D. 1967. Extraordinary sex ratios: a sex-ratio theory for sex linkage and inbreeding has new implications in cytogenetics and entomology. Science 156:477-488.

Iwasa, Y., and A. Pomiankowski. 2001. The evolution of X-linked genomic imprinting. Genetics 158:1801-1809.

Kondoh, M., and M. Higashi. 2000. Reproductive isolation mechanism resulting from resolution of intragenomic conflict. American Naturalist 156:511-518.

Lawson Handley, L. J., and N. Perrin. 2007. Advances in our understanding of mammalian sex-biased dispersal. Molecular Ecology 16:1559-1578.

Lehmann, L., F. Rousset, D. Roze, and L. Keller. 2007. Strong reciprocity or strong ferocity? a population genetic view of the evolution of altruistic punishment. American Naturalist 170:21-36.

Mills, W., and T. Moore. 2004. Polyandry, life-history trade-offs and the evolution of imprinting at Mendelian loci. Genetics 168:23172327.

Mochizuki, A., Y. Takeda, and Y. Iwasa. 1996. The evolution of genomic imprinting. Genetics 144:1283-1295.

Morison, I. M., J. P. Ramsay, and H. G. Spencer. 2005. A census of mammalian imprinting. Trends in Genetics 21:457-465.

Ogawa, O., L. A. McNoe, M. R. Eccles, I. M. Morison, and A. E. Reeve. 1993. Human insulin-like growth factor type I and type II receptors are not imprinted. Human Molecular Genetics 2:21632165.

Queller, D. C. 1994. Genetic relatedness in viscous populations. Evolutionary Ecology 8:70-73.

Rousset, F. 2004. Genetic structure and selection in subdivided populations. Princeton University Press, Princeton, NJ.

Rousset, F., and S. Billiard. 2000. A theoretical basis for measures of kin selection in subdivided populations: finite populations and localized dispersal. Journal of Evolutionary Biology 13:814-825.
Rousset, F., and D. Roze. 2007. Constraints on the origin and maintenance of genetic kin recognition. Evolution 61:2320-2330.

Roze, D., and F. Rousset. 2004. The robustness of Hamilton's rule with inbreeding and dominance: kin selection and fixation probabilities under partial sib mating. American Naturalist 164:214231.

2008. Multilocus models in the infinite island model of population structure. Theoretical Population Biology 73:529-542. Spencer, H. G. 2000. Population genetics and evolution of genomic imprinting. Annual Review of Genetics 34:457-477.

Spencer, H. G., and A. G. Clark. 2006. A chip off the old block: a model for the evolution of genomic imprinting via selection for parental similarity. Genetics 174:931-935.

Spencer, H. G., M. W. Feldman, and A. G. Clark. 1998. Genetic conflicts, multiple paternity and the evolution of genomic imprinting. Genetics 148:893-904.

Spencer, H. G., M. W. Feldman, A. G. Clark, and A. E. Weisstein. 2004. The effect of genetic conflict on genomic imprinting and modification of expression at a sex-linked locus. Genetics 166:565579 .

Taylor, P. D. 1990. Allele-frequency change in a class-structured population. American Naturalist 135:95-106.

1992. Altruism in viscous populations: an inclusive fitness model. Evolutionary Ecology 6:352-356.

Taylor, P. D., and S. A. Frank. 1996. How to make a kin selection model. Journal of Theoretical Biology 180:27-37.

Úbeda, F. 2008. Evolution of genomic imprinting with biparental care: implications for Prader-Willi and Angelman syndromes. PLoS Biology 6:e208.

Úbeda, F., and A. Gardner. Forthcoming. A model for genomic imprinting in the social brain: juveniles. Evolution, doi:10.1111/ j.1558-5646.2010.010105.x.

Van Cleve, J., and M. W. Feldman. 2007. Sex-specific viability, sex linkage and dominance in genomic imprinting. Genetics 176:11011118.

Wang, X., Q. Sun, S. D. McGrath, E. R. Mardis, P. D. Soloway, and A. G. Clark. 2008. Transcriptome-wide identification of novel imprinted genes in neonatal mouse brain. PLoS ONE 3:e3839.

Wild, G., and A. Traulsen. 2007. The different limits of weak selection and the evolutionary dynamics of finite populations. Journal of Theoretical Biology 247:382-390.

Wilkins, J. F., and D. Haig. 2003. What good is genomic imprinting: the function of parent-specific gene expression. Nature Reviews Genetics 4:359-368.

Wolf, J. B., and R. Hager. 2006. A maternal-offspring coadaptation theory for the evolution of genomic imprinting. PLoS Biology 4: e380.

Wright, S. 1931. Evolution in Mendelian populations. Genetics 16: 97-159.

Associate Editor: Peter D. Taylor Editor: Ruth G. Shaw 


\title{
Appendix from J. Van Cleve et al., "How Demography, Life History, and Kinship Shape the Evolution of Genomic Imprinting"
}

\author{
(Am. Nat., vol. 176, no. 4, p. 000)
}

\section{Methods}

\section{Fitness Functions}

In order to guarantee that the expected fitness function in equation (3) is correct to first order in $\delta_{x}$ and $\delta_{y}$, we define the $W$ functions in a mechanistic way. In what follows, we assume that expected values, E[·], are taken over all events in the life cycle subject to stochasticity, for example, mating, fertility, and density-independent survival. The only exception is that we ignore demographic stochasticity, and we assume that the population deterministically maintains a constant number of males and females in each generation. Let the random number of individuals of sex $v$ produced by parent $j$ of sex $u$ in deme $i$ be denoted by $\omega_{u v i j}$ (where $u$ and $v$ are each either $\mathrm{f}$ or $\mathrm{m}$ ) and the expected value of $\omega_{u v i j}$ be given by $\mathrm{E}\left[\omega_{u v i j}\right]=W_{u v i j}$. Let $R_{i j n}$ be an indicator random variable that is 1 when offspring $n$ of female $j$ in deme $i$ is male and 0 when that offspring is female. Since we do not allow the sex ratio to evolve, the probability that an offspring is male is $\mathrm{E}\left[R_{i j n}\right]=r$. Female offspring $n$ of female $j$ survives when the indicator variable $\sigma_{\mathrm{fijn}}$ is 1 and dies when it is 0 . The expected value of $\sigma_{\mathrm{f} i j n}$ is the survival probability for such an individual, namely, $s_{\mathrm{f}}\left(z_{i j n}\right)$. Likewise, $\sigma_{\mathrm{m} i j n}$ measures male offspring survival and $\mathrm{E}\left[\sigma_{\mathrm{m} i j n}\right]=s_{\mathrm{m}}\left(z_{i j n}\right)$. Finally, $\sigma_{\mathrm{f} i j}$ measures survival of adult female $j$ in deme $i$ with $\mathrm{E}\left[\sigma_{\mathrm{f} i j}\right]=s_{\mathrm{f}}\left(z_{i j}\right)$, and $\sigma_{\mathrm{m} i j}$ is defined similarly for adult males. Note that this notation for survival differs from that in the main text, where $S$ denotes the expected survival probability. Migration is tracked by the indicator random variable $M_{i j n \rightarrow i}$, which is 1 when offspring $n$ of female $j$ in deme $i$ migrates to deme $\ddot{i}$ and 0 when that offspring does not migrate to deme $\ddot{i}$. When $i \neq \ddot{i}, \mathrm{E}\left[M_{i j n \rightarrow i}\right]=m /(D-1)$ and $\mathrm{E}\left[M_{i j n \rightarrow i}\right]=1-m$. The first term in the fitness function is the survival of the focal adult. The second term accounts for offspring that replace dying adults, and because we assume that offspring survive independently of one another during density-dependent regulation, we can calculate this second term as the sum of ratios of the number of surviving offspring of a given sex that migrate to a particular deme divided by the total number of offspring that migrate to that deme; that is, for the (random) number of female offspring produced by a female, $\omega_{\mathrm{ffi} i j}$, we get

$$
\omega_{\mathrm{ff} i j}=2 \sigma_{\mathrm{f} i j}+\sum_{i=1}^{D} \frac{\left(N_{\mathrm{f}}-\sum_{j=1}^{N_{\mathrm{f}}} \sigma_{\mathrm{f} i j}\right) \sum_{n=1}^{K_{i j}} M_{i j n \rightarrow i}\left(1-R_{i j n}\right) \sigma_{\mathrm{f} i j n}}{\sum_{\hat{i}=1}^{D} \sum_{\hat{j}=1}^{N_{\mathrm{f}}} \sum_{n=1}^{K_{\hat{i}}^{\hat{j}}} M_{\hat{j} \hat{j} n \rightarrow i}\left(1-R_{\hat{i j n}}\right) \sigma_{\mathrm{f} \hat{i} \hat{j} n}},
$$

where the factor of 2 before $\sigma_{\mathrm{f} i j}$ accounts for the fact that a surviving parent "inherits" twice the number of alleles it donates to any offspring. The number of males produced by a focal female, $\omega_{\text {fmij }}$, is defined similarly, with the survival and sex-ratio indicator variables replaced by their male counterparts, the female population size $N_{\mathrm{f}}$ replaced by male population size $N_{\mathrm{m}}$, and the removal of the first term $\sigma_{\mathrm{f} i j}$. In order to define male fitness, we introduce another indicator variable, $L_{i \hat{j} \times \times j}$, which is 1 when offspring $n$ of female $\hat{j}$ in deme $i$ was fathered by male $j$ and 0 otherwise. To count the number of offspring of a focal male, we sum over all offspring of all females, using $L_{i \hat{j} n \times j}$. For example, the number of females produced by a focal male is

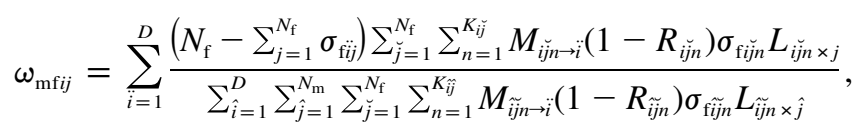

and $\omega_{\mathrm{mm} i j}$ is defined analogously by replacing the appropriate sex ratio and population variables, except that it has a leading term of $2 \sigma_{\mathrm{m} i j}$. It is important to note that both the numerator and the denominator of all the $W$ functions contain sums over the number of offspring of a given female before migration, $K_{i j}$, which is a random 
variable whose mean depends on the distribution of genotype frequencies in the population. In general, calculating $\mathrm{E}\left[\omega_{u v i j}\right]=W_{u v i j}$ is difficult because each fitness function is a ratio of random variables. However, since we assume that female fertility $\mathrm{E}\left[K_{i j}\right]$ is large, we can use the delta method (Rice 1995) to approximate the expected value of the ratio of random variables by the ratio of the expected values. This approximation assumes that the population sizes of females and males remain constant and that the effect of demographic stochasticity is negligible and is correct to order $1 / \mathrm{E}\left[K_{i j}\right]^{2}$. Thus, we can approximate $W_{\mathrm{ffij}}$, using equation (A1), as

$$
\begin{aligned}
& W_{\mathrm{ffi} j} \approx 2 \mathrm{E}\left[\sigma_{\mathrm{f} i j}\right]+\left\{\frac{\left(N_{\mathrm{f}}-\sum_{j=1}^{N_{\mathrm{f}}} \mathrm{E}\left[\sigma_{\mathrm{f} i j}\right]\right)(1-m) \mathrm{E}\left[\sum_{n=1}^{K_{i j}} \sigma_{\mathrm{f} i j n}\right]}{(1-m) \sum_{\hat{j}=1}^{N_{\mathrm{f}}} \mathrm{E}\left[\sum_{n=1}^{K_{\hat{j}}} \sigma_{\mathrm{f} \hat{j} \hat{j} n}\right]+[m /(D-1)] \sum_{\hat{i} \neq i}^{D} \sum_{\hat{j}=1}^{N_{\mathrm{f}}} \mathrm{E}\left[\sum_{n=1}^{K_{\hat{i}}} \sigma_{\mathrm{f} \hat{j} \hat{j} n}\right]}\right.
\end{aligned}
$$

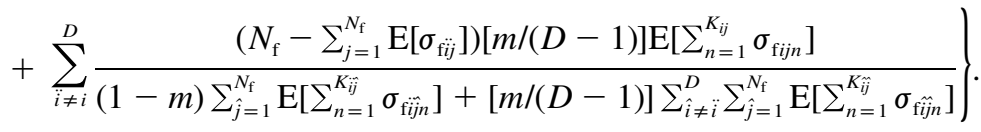

This expression can be simplified as

$$
W_{\mathrm{ffij}} \approx 2 S_{\mathrm{fi} i j}+\frac{\left(1-S_{\mathrm{fi}}\right)(1-m) J_{\mathrm{ffi} i j}}{(1-m) J_{\mathrm{ffi} i}+m J_{\mathrm{ff}}}+\frac{\left(1-S_{\mathrm{f}}\right) m J_{\mathrm{ffi} i j}}{J_{\mathrm{ff}}}
$$

where $S_{\mathrm{fij}}=\mathrm{E}\left[\sigma_{\mathrm{fij}}\right]$ is the expected survival probability of female adult $j$ and the expected number of females juveniles produced before migration by a female, $\mathrm{E}\left[\sum_{n=1}^{K_{i j}} \sigma_{\mathrm{fij} n}\right]$, is denoted by $J_{\mathrm{ffij}}$. The average adult survival probability for females in deme $i$ is $S_{\mathrm{fi}}=\left(1 / N_{\mathrm{f}}\right) \sum_{j=1}^{N_{\mathrm{f}}} \mathrm{E}\left[\sigma_{\mathrm{fij}}\right]$, and the average across all demes is $S_{\mathrm{f}}=$ $(1 / D) \sum_{i=1}^{D} S_{\mathrm{f} i}$. The average of number of female juveniles produced by females in deme $i$ is computed similarly and is denoted by $J_{\mathrm{ffi}}$, and the average across all demes is denoted by $J_{\mathrm{ff}}$. The analogous expression for the number of females produced by a male, $W_{\mathrm{mfij}}$, can be approximated as

$$
W_{\mathrm{mf} i j} \approx \frac{\left(1-S_{\mathrm{fi}}\right)(1-m) J_{\mathrm{mf} i j}}{(1-m) J_{\mathrm{mf} i}+m J_{\mathrm{mf}}}+\frac{\left(1-S_{\mathrm{f}}\right) m J_{\mathrm{mf} i j}}{J_{\mathrm{mf}}}
$$

where

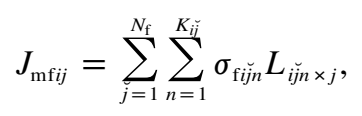

$J_{\mathrm{mf} i}$ is the average of $J_{\mathrm{mfij}}$ in males in deme $i$, and $J_{\mathrm{mf}}$ is the average of $J_{\mathrm{mf} i j}$ across all demes. Equations (A4) and (A5), along with similar approximations for the expected values of $W_{\mathrm{fm} i j}$ and $W_{\mathrm{mm} i j}$, generate equation (3).

\section{Expected Number of Juveniles before Migration}

Here, we will show that the expressions for the expected number of juveniles of sex $v$ produced before migration by females and males, $J_{f v i j}$ and $J_{m i j}$, in equations (4) and (5), respectively, are correct to first order in $\delta_{x}$ and $\delta_{y}$. We begin by deriving a first-order expression for $J_{f_{v i j}}=\mathrm{E}\left[\sum_{n=1}^{K_{i j}} \sigma_{v i j n}\right]$ from the underlying assumptions about female fertility, offspring survival, and mating given in "Model Framework." Note that $\mathrm{E}\left[\sum_{n=1}^{K_{i j}} \sigma_{v i j n}\right]$ is a random sum, since $K_{i j}$ depends on the genotypes of the males that mate with a female during random mating and on which alleles are donated to the offspring during meiotic segregation. The survival component, $\sigma_{v i j n}$, also depends on parental genotypes through the offspring genotype. In short, the fertility-viability trade-off results in nonindependence between maternal fertility and offspring survival. This means that we cannot evaluate the expected value of the sum simply as the product of the expected fertility and the expected survival. Rather, we first condition the expectation on the frequency of $A$, denoted by $T$, donated by the males mating with a focal female who mates $\tau$ times; using this conditioning, 


$$
\mathrm{E}\left[\sum_{n=1}^{K_{i j}} \sigma_{v i j n}\right]=\sum_{t=0}^{\tau} \mathrm{E}\left[\sum_{n=1}^{K_{i j}} \sigma_{v i j n} \mid T=\frac{t}{\tau}\right] \operatorname{Pr}\left[T=\frac{t}{\tau}\right],
$$

where $t$ is the number of $A$ alleles donated by males. Once the maternally derived allele frequency $p_{\mathrm{fi} j}$ and the paternally derived allele frequency $t / \tau$ are fixed, the fertility $K_{i j}$ and survival $\sigma_{v i j n}$ are independent. This is because we assume that female fertility is a function of the mean phenotype in the offspring, $z\left(p_{\mathrm{fij}}, t / \tau\right)$, which is fixed once we condition on the maternally and paternally derived allele frequencies. Thus, we can write the right-hand side of equation (A6) as

$$
\sum_{t=0}^{\tau} \mathrm{E}\left[K_{i j} \mid T=\frac{t}{\tau}\right] \mathrm{E}\left[\sigma_{v i j n} \mid T=\frac{t}{\tau}\right] \operatorname{Pr}\left[T=\frac{t}{\tau}\right]
$$

The expected fertility $\mathrm{E}\left[K_{i j} \mid T=t / \tau\right]$ is just $b\left(z\left(p_{\mathrm{fij}}, t / \tau\right)\right)$, and the expected survival $\mathrm{E}\left[\sigma_{\mathrm{fij} i} \mid T=t / \tau\right]$ is given by

$$
p_{\mathrm{fij}}\left(\frac{t}{\tau}\right) s_{v}\left(z_{A A}\right)+p_{\mathrm{fij}}\left(1-\frac{t}{\tau}\right) s_{v}\left(z_{A a}\right)+\left(1-p_{\mathrm{fij}}\right)\left(\frac{t}{\tau}\right) s_{v}\left(z_{a A}\right)+\left(1-p_{\mathrm{fij}}\right)\left(1-\frac{t}{\tau}\right) s_{v}\left(z_{a a}\right)
$$

The distribution of $T$ is binomial, with parameters $\tau$ and $p_{\text {mi }}$, since females select each of their $\tau$ mates randomly with replacement and since meiotic segregation is fair. Substituting the expected survival and fertility and the probability mass function for $T$ into equation (A7) yields

$$
\begin{aligned}
J_{\mathrm{f} v i j}= & \sum_{t=0}^{\tau}\left(\begin{array}{l}
\tau \\
t
\end{array}\right) p_{\mathrm{m} i}^{t}\left(1-p_{\mathrm{m} i}\right)^{\tau-t} b\left(z\left(p_{\mathrm{fij}}, \frac{t}{\tau}\right)\right) \\
& \times\left[p_{\mathrm{fij} i}\left(\frac{t}{\tau}\right) s_{v}\left(z_{\mathrm{AA}}\right)+p_{\mathrm{fij}}\left(1-\frac{t}{\tau}\right) s_{v}\left(z_{\mathrm{Aa}}\right)+\left(1-p_{\mathrm{fij}}\right)\left(\frac{t}{\tau}\right) s_{v}\left(z_{a A}\right)+\left(1-p_{\mathrm{fij}}\right)\left(1-\frac{t}{\tau}\right) s_{v}\left(z_{a a}\right)\right] .
\end{aligned}
$$

The expected number of juveniles of sex $v$ produced by a male before migration, $J_{m v i j}$, is more complex, since we must account for the variable level of paternity that a focal male can have in the brood of any particular female. Deriving $J_{m v i j}$ follows a logic similar to that for the derivation of equation (A8), except that we first condition on the number of times male $j$ mates with female $\breve{j}$, which we denote $G$ (note that since mating is random, $G$ does not depend on the genotypes of either males or females). We then condition on the frequency of $A$ donated by males mating with female $j$, except that now this frequency depends on fraction of the total paternity that male $j$ contributes to the offspring of female $\breve{j}$; we denote this frequency $T(G)$ to indicate the dependence on $G$. Conditioning $J_{\mathrm{m} v i j}=\mathrm{E}\left[\sum_{\tilde{j}=1}^{N_{\mathrm{f}}} \sum_{n=1}^{K_{i \check{j}}} \sigma_{v i \check{n} n} L_{i \check{j} n \times j}\right]$ on $G$ and $T(G)$ yields

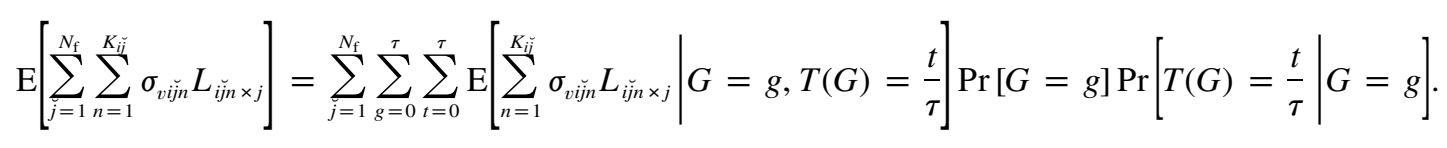

Once we have conditioned on $G$ and $T(G)$, the fertility of female $\breve{j}, K_{i j}$, is independent of whether the offspring survives, $\sigma_{v i \grave{n} n}$, and whether male $j$ fathered the offspring, $L_{i \check{i} \times \times j}$, which means that

$$
\mathrm{E}\left[\sum_{n=1}^{K_{\check{i ̆}}} \sigma_{v \check{\check{j} n}} L_{i \check{i j n} \times j} \mid G=g, T(G)=\frac{t}{\tau}\right]=E\left[K_{\check{i j}} \mid G=g, T(G)=\frac{t}{\tau}\right] \mathrm{E}\left[\sigma_{v i \check{j} n} L_{\check{i j n} \times j} \mid G=g, T(G)=\frac{t}{\tau}\right] .
$$

As above, $\mathrm{E}\left[K_{i j} \mid G=g, T(G)=t / \tau\right]=b\left(z\left(p_{\mathrm{f} i j ;}, t / \tau\right)\right)$. The second expectation on the right-hand side of equation (A10) is the probability that offspring $n$ both survives and is fathered by male $j$ and can be written as 


$$
\begin{aligned}
\mathrm{E}\left[\sigma_{v i \grave{i j n}} L_{\check{i j n} \times j} \mid G=g, T(G)=\frac{t}{\tau}\right]=\frac{g}{\tau} & {\left[p_{\mathrm{f} i j} p_{\mathrm{m} i j} s_{v}\left(z_{A A}\right)+p_{\mathrm{fij}}\left(1-p_{\mathrm{m} i j}\right) s_{v}\left(z_{A a}\right)\right.} \\
& \left.+\left(1-p_{\mathrm{f} i j}\right) p_{\mathrm{m} i j} s_{v}\left(z_{a A}\right)+\left(1-p_{\mathrm{fij}}\right)\left(1-p_{\mathrm{m} i j}\right) s_{v}\left(z_{a a}\right)\right] .
\end{aligned}
$$

Here, $g / \tau$ is the fraction of the total paternity that male $j$ contributes to the offspring of female $\breve{j}$ and is the probability that any particular offspring was fathered by male $j$. The term in brackets on the right-hand side of equation (A11) is the probability that an offspring of male $j$ and female $\breve{j}$ survives.

The remaining components to be calculated in equation (A9) are the probability functions $G$ and $T(G)$. Since mating is random, $G$ is binomial with parameters $\tau$ and $1 / N_{\mathrm{m}}$. As described above for female reproductive output, $T(G)$ is binomial but now with parameters $\tau$ and $\rho_{\mathrm{m} i}(j, g)$, which is the frequency of $A$ donated by males mating with a specific female, given that male $j$ mates with that female $g$ times, and is given by

$$
\rho_{\mathrm{m} i}(j, g)=\left(\frac{g}{\tau}\right) p_{m i j}+\left(1-\frac{g}{\tau}\right) \frac{\left(N_{\mathrm{m}} p_{\mathrm{m} i}-p_{\mathrm{m} i j}\right)}{N_{\mathrm{m}}-1}
$$

Substituting equations (A10) and (A11) and the probability functions for $G$ and $T(G)$ into equation (A9) yields

$$
\begin{aligned}
& J_{\mathrm{m} v i j}=\sum_{j=1}^{N_{\mathrm{f}}} \sum_{g=0}^{\tau}\left(\begin{array}{l}
\tau \\
g
\end{array}\right)\left(\frac{1}{N_{\mathrm{m}}}\right)^{g}\left(1-\frac{1}{N_{\mathrm{m}}}\right)^{\tau-g} \frac{g}{\tau} \sum_{t=0}^{\tau}\left(\begin{array}{l}
\tau \\
t
\end{array}\right) \rho_{\mathrm{m} i}(j, g)^{t}\left(1-\rho_{\mathrm{m} i}(j, g)\right)^{\tau-t} b\left(z\left(p_{\mathrm{fij}}, \frac{t}{\tau}\right)\right) \\
& \times\left[p_{\mathrm{f} i j j} p_{\mathrm{m} i j} s_{v}\left(z_{A A}\right)+p_{\mathrm{f} i j}\left(1-p_{\mathrm{m} i j}\right) s_{v}\left(z_{A a}\right)+\left(1-p_{\mathrm{f} i j}\right) p_{\mathrm{m} i j} s_{v}\left(z_{a A}\right)+\left(1-p_{\mathrm{fij}}\right)\left(1-p_{\mathrm{m} i j}\right) s_{v}\left(z_{a a}\right)\right] .
\end{aligned}
$$

Using the definition of $z$ given in equation (1), we can expand equation (A8) to first order, which produces

$$
\begin{aligned}
J_{\mathrm{f} v i j}= & b\left(z_{a a}\right) s_{v}\left(z_{a a}\right) \\
& \times\left(1+\left(B_{v}-C\right)\left\{2(1-h)\left(p_{\mathrm{fij}} \delta_{x}+p_{\mathrm{m} i} \delta_{y}\right)+[1-2(1-h)] p_{\mathrm{fij}} p_{\mathrm{m} i}\left(\delta_{x}+\delta_{y}\right)\right\}\right)+O\left(\delta^{2}\right),
\end{aligned}
$$

where the functions $b$ and $s_{v}$ are evaluated at $z_{a a}$ and $\delta$ is the larger of $\delta_{x}$ and $\delta_{y}$. Equation (A14) is identical to first order to the expression given for $J_{f v i j}$ in equation (4). Likewise, a first-order analysis of equation (A13) shows that

$$
\begin{aligned}
J_{\mathrm{m} v i j}=\frac{N_{\mathrm{f}}}{N_{\mathrm{m}}} b\left(z_{a a}\right) s_{v}\left(z_{a a}\right) & \\
\times & {\left[1+\left(B_{v}-\frac{1}{\tau} C\right)\left(\left\{2(1-h)\left(p_{\mathrm{fi}} \delta_{x}+p_{\mathrm{m} i j} \delta_{y}\right)+[1-2(1-h)] p_{\mathrm{fi}} p_{\mathrm{m} i j}\left(\delta_{x}+\delta_{y}\right)\right\}\right.\right.} \\
& \left.\left.\quad\left(1-\frac{1}{\tau}\right) C\left\{2(1-h)\left(p_{\mathrm{fi}} \delta_{x}+p_{\mathrm{m} i} \delta_{y}\right)+[1-2(1-h)] p_{\mathrm{fi}} p_{\mathrm{m} i}\left(\delta_{x}+\delta_{y}\right)\right\}\right)\right]+O\left(\delta^{2}\right),
\end{aligned}
$$

which is identical to first order to the formula for $J_{m v i j}$ in equation (5).

\section{Selection Gradients and Relatedness}

Using the expressions for the fitness function $W_{u v i j}$ in equation (3), the formula for $\Delta p$ in equation (2), the number of juveniles produced by females and males in equations (4) and (5), respectively, and the definition for the gene expression phenotype $z$ in equation (1), we first show how to derive the expressions for the selection gradients $\mathcal{S}_{x}$ and $\mathcal{S}_{y}$ in equations (7) and (8), respectively, which are correct to first order in $\delta_{x}$ and $\delta_{y}$, and then 
provide expressions for the relatedness coefficients that appear in $\mathcal{S}_{x}$ and $\mathcal{S}_{y}$ in terms of probabilities of genetic identity.

Beginning with the adult-survival component in the fitness function in equation (3), we note that the expected survival probability of adult $j$ of $\operatorname{sex} u$ in deme $i$ is $\mathrm{E}\left[\sigma_{u i j}\right]=s_{u}\left(z\left(p_{u \mathrm{f} i j}, p_{u \mathrm{~m} i j}\right)\right)$, where $p_{u \mathrm{f} i j}$ is the frequency of $A$ in the maternally derived allele of the focal adult and $p_{u \mathrm{~m} i j}$ is the frequency of $A$ in the paternally derived allele. Using equation (1), we can then calculate $\mathrm{E}\left[\sigma_{u i j}\right]$ to first order in $\delta_{x}$ and $\delta_{y}$ as

$$
\mathrm{E}\left[\sigma_{u i j}\right]=s_{u}+s_{u} B_{u}\left\{2(1-h)\left(p_{u \mathrm{f} i j} \delta_{x}+p_{u \mathrm{~m} i j} \delta_{y}\right)+[1-2(1-h)] p_{u \mathrm{f} i j} p_{u \mathrm{~m} i j}\left(\delta_{x}+\delta_{y}\right)\right\}
$$

Averaging equation (A16) over all individuals of sex $u$ in a deme and over all individuals of sex $u$ in the metapopulation yields

$$
\mathrm{E}\left[\sigma_{u i}\right]=s_{u}+s_{u} B_{u}\left\{2(1-h)\left(p_{u \mathrm{fi} i} \delta_{x}+p_{u \mathrm{~m} i} \delta_{y}\right)+[1-2(1-h)] \overline{p_{u \mathrm{f} i j} p_{u \mathrm{~m} i j}}\left(\delta_{x}+\delta_{y}\right)\right\}
$$

and

$$
\mathrm{E}\left[\sigma_{u}\right]=s_{u}+s_{u} B_{u}\left\{2(1-h)\left(p_{u \mathrm{f}} \delta_{x}+p_{u \mathrm{~m}} \delta_{y}\right)+[1-2(1-h)] \overline{p_{u \mathrm{f} i j} p_{u \mathrm{~m} i j}}\left(\delta_{x}+\delta_{y}\right)\right\}
$$

respectively. Plugging the adult survival probabilities from equations (A16)-(A18) into the fitness function in equation (3) and evaluating the whole expression to first order yields three components. The first component is the zeroth-order term, which for $W_{u u i j}$ and $W_{u \hat{u} i j}$ are $1+s_{u}$ and $1-s_{\hat{u}}$, respectively (the hat denotes a complement; i.e., $\hat{f}=m$ and $\hat{m}=f$ ). These terms cancel out with $p=\alpha_{\mathrm{f}} p_{\mathrm{f}}+\alpha_{\mathrm{m}} p_{\mathrm{m}}$ in the equation for $\Delta p$. The second component is the product of the zeroth-order terms for adult survival, $1-s_{u}$ for $W_{u i j j}$ and $1-s_{\hat{u}}$ for $W_{u \hat{u} i j}$, and the first-order terms for juveniles surviving to adulthood (which are the first-order terms times $\beta$ from eq. [3]). After substituting the second-component terms into the formula for $\Delta p$ in equation (2), we find that they are weighted by the reproductive values $\alpha_{\mathrm{f}}$ and $\alpha_{\mathrm{m}}$. Since

$$
\alpha_{\mathrm{f}}\left(1-s_{\mathrm{f}}\right)=\frac{\left(1-s_{\mathrm{f}}\right)\left(1-s_{\mathrm{m}}\right)}{2-s_{\mathrm{f}}-s_{\mathrm{m}}}=\alpha_{\mathrm{m}}\left(1-s_{\mathrm{m}}\right)=\beta
$$

these second-component terms contribute

$$
\beta\left[B_{\mathrm{f}} R_{j s, \mathrm{ff}}+B_{\mathrm{m}} R_{j s, \mathrm{mf}}-C\left(R_{b, \mathrm{ff}}+R_{b, \mathrm{mf}}\right)\right]
$$

to $\mathcal{S}_{x}$ and

$$
\beta\left[B_{\mathrm{f}} R_{j s, \mathrm{fm}}+B_{\mathrm{m}} R_{j s, \mathrm{~mm}}-C\left(R_{b, \mathrm{fm}}+R_{b, \mathrm{~mm}}\right)\right]
$$

to $\mathcal{S}_{y}$. The relatedness coefficients $R_{a s, u v}, R_{j s, u v}$, and $R_{b, u v}$ that arise from this process are given by

$$
\begin{aligned}
R_{j s, u v}= & (1-h)\left[\overline{p_{v i j}^{2}}-p_{v}^{2}+\overline{p_{v i} p_{\hat{v} i}}-p_{v} p_{\hat{v}}-\left(1-m_{u}\right)^{2}\left(\overline{p_{v i}^{2}}-p_{v}^{2}+\overline{p_{v i} p_{\hat{v} i}}-p_{v} p_{\hat{v}}\right)\right] \\
& +\frac{1}{2}[1-2(1-h)] R_{j s, u h}, \\
R_{b, u \mathrm{f}}= & (1-h)\left[\overline{p_{f i j}^{2}}-p_{f}^{2}+\overline{p_{f i} p_{m i}}-p_{f} p_{m}-\left(1-m_{u}\right)^{2}\left(\overline{p_{\mathrm{f} i}^{2}}-p_{\mathrm{f}}^{2}+\overline{p_{\mathrm{f} i} p_{\mathrm{m} i}}-p_{\mathrm{f}} p_{\mathrm{m}}\right)\right] \\
& +\frac{1}{2}[1-2(1-h)] R_{b, u h}, \\
R_{b, u \mathrm{~m}}= & (1-h)\left[\frac{1}{\tau} \overline{p_{\mathrm{m} i j}^{2}}+\left(1-\frac{1}{\tau}\right) \overline{p_{\mathrm{m} i}^{2}}-p_{\mathrm{m}}^{2}+\overline{p_{\mathrm{f} i} p_{\mathrm{m} i}}-p_{\mathrm{f}} p_{\mathrm{m}}-\left(1-m_{u}\right)^{2}\left(\overline{p_{\mathrm{m} i}^{2}}-p_{\mathrm{m}}^{2}+\overline{p_{\mathrm{fi} i} p_{\mathrm{m} i}}-p_{\mathrm{f}} p_{\mathrm{m}}\right)\right] \\
& +\frac{1}{2}[1-2(1-h)] R_{b, u h},
\end{aligned}
$$


where

$$
R_{j s, u h}=\overline{p_{\mathrm{f} i j}^{2} p_{\mathrm{m} i}}-\overline{p_{\mathrm{fi} i} p_{\mathrm{m} i}} p_{\mathrm{f}}+\overline{p_{\mathrm{m} i j}^{2} p_{\mathrm{f} i}}-\overline{p_{\mathrm{fi} i} p_{\mathrm{m} i}} p_{\mathrm{m}}-\left(1-m_{u}\right)^{2}\left(\overline{p_{\mathrm{f} i}^{2} p_{\mathrm{m} i}}-\overline{p_{\mathrm{fi} i} p_{\mathrm{m} i}} p_{\mathrm{f}}+\overline{p_{\mathrm{m} i}^{2} p_{\mathrm{f} i}}-\overline{p_{\mathrm{fi} i} p_{\mathrm{m} i}} p_{\mathrm{m}}\right)
$$

and

$$
\begin{aligned}
R_{b, u h}= & \overline{p_{\mathrm{f} i j}^{2} p_{\mathrm{m} i}}-\overline{p_{\mathrm{f} i} p_{\mathrm{m} i}} p_{\mathrm{f}}+\frac{1}{\tau} \overline{p_{\mathrm{f} i} p_{\mathrm{m} i j}^{2}}+\left(1-\frac{1}{\tau}\right) \overline{p_{\mathrm{fi} i} p_{\mathrm{m} i}^{2}}-\overline{p_{\mathrm{fi} i} p_{\mathrm{m} i}} p_{\mathrm{m}} \\
& -\left(1-m_{u}\right)^{2}\left(\overline{p_{\mathrm{fi} i}^{2} p_{\mathrm{m} i}}-\overline{p_{\mathrm{f} i} p_{\mathrm{m} i}} p_{\mathrm{f}}+\overline{p_{\mathrm{fi} i} p_{\mathrm{m} i}^{2}}-\overline{p_{\mathrm{fi} i} p_{\mathrm{m} i}} p_{\mathrm{m}}\right) .
\end{aligned}
$$

The overline represents an average of the given product of allele frequencies over all demes. The products of allele frequencies can be viewed as probabilities of genetic identity. For example, $\overline{p_{\mathrm{fij}}^{2}}$ is the probability that two alleles drawn with replacement from the same female are both $A$. Likewise, $\overline{p_{\mathrm{fi}}^{2} p_{\mathrm{m} i}}$ is the probability that three alleles from the same deme, two alleles drawn from females with replacement and one allele from a male, are all A.

The third component of the fitness functions is a product of the zeroth-order terms for juveniles surviving to adulthood and the first-order terms of adult survival. These final components are

$$
\begin{aligned}
s_{u} B_{u} & \left(2(1-h)\left(p_{u \mathrm{fij}} \delta_{x}+p_{u \mathrm{~m} i j} \delta_{y}\right)+[1-2(1-h)] p_{u \mathrm{fij}} p_{u \mathrm{mij} i j}\left(\delta_{x}+\delta_{y}\right)\right. \\
& +(1-m)\left\{2(1-h)\left(p_{u f i} \delta_{x}+p_{u \mathrm{~m} i} \delta_{y}\right)+[1-2(1-h)] \overline{p_{u \mathrm{fi} i j} p_{u \mathrm{~m} i j}}\left(\delta_{x}+\delta_{y}\right)\right\} \\
& \left.+m\left\{2(1-h)\left(p_{u \mathrm{f}} \delta_{x}+p_{u \mathrm{~m}} \delta_{y}\right)+[1-2(1-h)] \overline{p_{u \mathrm{fij} i} p_{u \mathrm{~m} i j}}\left(\delta_{x}+\delta_{y}\right)\right\}\right)
\end{aligned}
$$

and

$$
\begin{aligned}
& s_{\hat{u}} B_{\hat{u}}(1-m)\left\{2(1-h)\left(p_{\hat{u} t i} \delta_{x}+p_{\hat{u} \mathrm{~m} i} \delta_{y}\right)+[1-2(1-h)] \overline{p_{\hat{u} \mathrm{t} i j} p_{\hat{u} \mathrm{mij}}}\left(\delta_{x}+\delta_{y}\right)\right\} \\
& \left.+m\left\{2(1-h)\left(p_{\hat{u} \mathrm{f}} \delta_{x}+p_{\hat{u} \mathrm{~m}} \delta_{y}\right)+[1-2(1-h)] \overline{p_{\hat{u} \mathrm{tij}} p_{\hat{u} \mathrm{~m} i j}}\left(\delta_{x}+\delta_{y}\right)\right\}\right)
\end{aligned}
$$

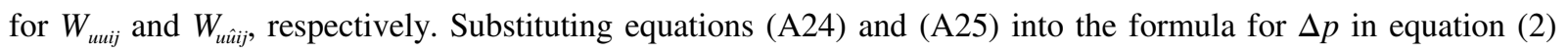
and simplifying, assuming that $h=1 / 2$, yields a contribution of

$$
\alpha_{\mathrm{f}} s_{\mathrm{f}} B_{\mathrm{f}} R_{a s, \mathrm{ff}}+\alpha_{\mathrm{m}} s_{\mathrm{m}} B_{\mathrm{m}} R_{a s, \mathrm{mf}}
$$

to $\mathcal{S}_{x}$ and

$$
\alpha_{\mathrm{f}} s_{\mathrm{f}} B_{\mathrm{f}} R_{a s, \mathrm{fm}}+\alpha_{\mathrm{m}} s_{\mathrm{m}} B_{\mathrm{m}} R_{a s, \mathrm{~mm}}
$$

to $\mathcal{S}_{y}$, where

$$
R_{a s, u v}=\frac{1}{4}\left[2 \overline{p_{u v i j}^{2}}+2 \overline{p_{u f i j} p_{u \mathrm{~m} i j}}-(1-m)\left(\overline{p_{u v i} p_{\mathrm{ffi} i}}+\overline{p_{u v i} p_{\mathrm{fm} i}}+\overline{p_{u v i} p_{\mathrm{mf} i}}+\overline{p_{u v i} p_{\mathrm{mm} i}}\right)-m p_{u v}\left(p_{\mathrm{ff}}+p_{\mathrm{fm}}+p_{\mathrm{mf}}+p_{\mathrm{mm}}\right)\right] .
$$

Combining the terms for $\mathcal{S}_{x}$ in equations (A19) and (A26) yields equation (7), and the terms for $\mathcal{S}_{y}$ in equations (A20) and (A27) yield equation (8).

\section{Probabilities of Identity by Descent}

In order to simplify our analysis of the genetic-identity values that appear in the relatedness coefficients presented in "Selection Gradients and Relatedness," we follow standard methods (Roze and Rousset 2003; Wakeley 2003) and assume that our model possesses the so-called separation of timescales property, which applies in the limit as $\delta \rightarrow 0$ and $D \rightarrow \infty$. In a metapopulation, two alleles sampled from different demes can 
coalesce only if they were in the same deme at some point in the past. The probability that these alleles came from the same deme in the past is of order $1 / D$, which goes to 0 as $D \rightarrow \infty$. Going backward in time, the ancestral lineages of two allele sampled in the same deme can either stay in the same deme and coalesce or move to different demes, in which case they take an infinite amount of time to return to the same deme and coalesce. Thus, coalescence occurs on two timescales, a fast timescale within demes and a slow timescale between demes. This assumption allows us to define the probability of identity by descent (IBD) of a set of alleles as the probability that the set of alleles coalesces in a finite number of generations within a deme before any lineage leaves the deme.

Since we evaluate the genetic identities under neutrality, the average allele frequencies over all demes in females and males at equilibrium will be equal; that is, $p_{\mathrm{f}}=p_{\mathrm{m}}=p$. The probability that two alleles drawn from the same female with replacement are identical is

$$
\overline{p_{\mathrm{f} i j}^{2}}=\frac{1}{2}\left(p+\overline{p_{\mathrm{ff} i j} p_{\mathrm{fm} i j}}\right),
$$

where $\overline{p_{\mathrm{ffij}} p_{\mathrm{fm} i j}}$ is the probability that the maternally and paternally derived alleles in a female are identical. The reasoning for equation (A29) is that we sample the same allele twice with probability $1 / 2$, in which case the alleles are both $A$ with probability $p$, and sample different alleles with probability $1 / 2$, in which case they are both $A$ with probability $\overline{p_{\mathrm{ff} i j} p_{\mathrm{fm} i j}}$. Let $Q_{u v}$ denote the probability that two alleles in different adults, one sampled from sex $u$ and one from $\operatorname{sex} v$, are identical by descent. We find that

$$
\overline{p_{\mathrm{ffij}} p_{\mathrm{fm} i j}}=Q_{\mathrm{fm}} p+\left(1-Q_{\mathrm{fm}}\right) p^{2}=p^{2}+Q_{\mathrm{fm}} p q=\overline{p_{\mathrm{m} i j} p_{\mathrm{mm} i j}},
$$

since the maternally and paternally derived alleles coalesce with probability $Q_{\mathrm{fm}}$ and are both $A$ with probability $p$ or fail to coalesce with probability $1-Q_{\mathrm{fm}}$ and are both $A$ with probability $p^{2}$. We note that in this model $Q_{\mathrm{fm}}$ is equal to the coefficient of inbreeding, $F$. This same reasoning shows that $\overline{p_{\mathrm{f} i j}^{2}}=\overline{p_{\mathrm{m} i j}^{2}}$ and yields

$$
\begin{aligned}
\overline{p_{\mathrm{f} i}^{2}} & =\frac{1}{2 N_{\mathrm{f}}}\left[p(1+p)+Q_{\mathrm{fm}} p q\right]+\left(1-\frac{1}{N_{\mathrm{f}}}\right)\left(p^{2}+Q_{\mathrm{ff}} p q\right), \\
\overline{p_{\mathrm{fi} i} p_{\mathrm{m} i}} & =p^{2}+p q Q_{\mathrm{fm}}, \\
\overline{p_{\mathrm{m} i}^{2}} & =\frac{1}{2 N_{\mathrm{m}}}\left[p(1+p)+Q_{\mathrm{fm}} p q\right]+\left(1-\frac{1}{N_{\mathrm{m}}}\right)\left(p^{2}+Q_{\mathrm{mm}} p q\right) .
\end{aligned}
$$

The remaining genetic identities in the relatedness coefficients are products of three allele frequency variables and appear only when $h \neq 1 / 2$; these identities are analyzed in "Dominance" below.

We now derive an equation for $Q_{u v}$. Let $Q^{\mathrm{J}}$ be the probability that two alleles are drawn from different juveniles. Since juveniles have not had a chance to undergo dispersal and viability selection, allele frequencies in juvenile males and females are the same and $Q^{\mathrm{J}}$ does not take sex-specific values. Also, let $C_{\mathrm{f} 21}$ denote the probability that two different maternally derived alleles drawn from different juveniles come from the same adult female in the previous generation; that is, $C_{\mathrm{f} 21}$ is the probability that two juveniles have the same mother, and likewise, $C_{\mathrm{m} 21}$ is the probability that two juveniles have the same father. Given the mating system described in "Model Framework,"

$$
\begin{aligned}
C_{\mathrm{f} 21} & =\frac{1}{N_{\mathrm{f}}}, \\
C_{\mathrm{m} 21} & =\frac{1}{N_{\mathrm{f}}}\left[\frac{1}{\tau}+\left(1-\frac{1}{\tau}\right) \frac{1}{N_{\mathrm{m}}}\right]+\left(1-\frac{1}{N_{\mathrm{f}}}\right) \frac{1}{N_{\mathrm{m}}} .
\end{aligned}
$$

At this point, we assume that migration is not sex specific and that generations are nonoverlapping; analyses of $Q_{u v}$ for these cases are given in "Sex-Specific Migration" and "Overlapping Generations" below, respectively. 
Since alleles can be identical by descent only if they come from the same deme under the infinite island model, $Q_{\mathrm{fm}}=Q_{\mathrm{ff}}=Q_{\mathrm{mm}}=(1-m)^{2} Q^{\mathrm{J}}$. The recursion for $Q^{\mathrm{J}}$ is

$$
Q^{\mathrm{J}}=\frac{1}{4}\left[C_{\mathrm{f} 21} \frac{1+Q_{\mathrm{fm}}}{2}+\left(1-C_{\mathrm{f} 21}\right) Q_{\mathrm{ff}}\right]+\frac{1}{2} Q_{\mathrm{fm}}+\frac{1}{4}\left[C_{\mathrm{m} 21} \frac{1+Q_{\mathrm{fm}}}{2}+\left(1-C_{\mathrm{m} 21}\right) Q_{\mathrm{mm}}\right]
$$

Substituting $Q_{\mathrm{fm}}$ appropriately into equation (A36) and simplifying yields

$$
Q_{\mathrm{fm}}=\frac{(1-m)^{2}[N(r+\tau)-1]}{(1-m)^{2}[N(r+\tau)-1]+8 m(2-m) \tau r(1-r) N^{2}},
$$

which is also given in equation (15). Equation (15) reduces to the standard formula for $F_{\mathrm{ST}}$ under random mating (Wang 1997); that is, when $\tau \rightarrow \infty$,

$$
Q_{\mathrm{fm}} \rightarrow \frac{(1-m)^{2}}{(1-m)^{2}+8 m(2-m) r(1-r) N}
$$

To get a sense for how multiple paternity affects $Q_{\mathrm{fm}}$, which measures the level of inbreeding, we approximate $Q_{\mathrm{fm}}$ under a local diffusion limit within demes where $N \rightarrow \infty, m \rightarrow 0$, and $N m \rightarrow M$. Under these conditions, equation (15) reduces to

$$
Q_{\mathrm{fm}}=\frac{r+\tau}{r+\tau+16 M r(1-r) \tau}
$$

In figure $\mathrm{A} 1$, we plot $Q_{\mathrm{fm}}$ relative to the value of $F_{\mathrm{ST}}$ for a randomly mating population under the local diffusion limit for $M=1$ and a range of values of $r$ and $\tau$. It is evident that the degree of multiple paternity $\tau$ has only a modest effect on the magnitude of $Q_{\mathrm{fm}}$, compared to the sex ratio, $r$; although not shown, $M$ has the largest effect on the magnitude of $Q_{\mathrm{fm}}$. Thus, it seems that increasing the number of times that a female mates $(\tau)$ has only a small effect on the total degree of population structure.

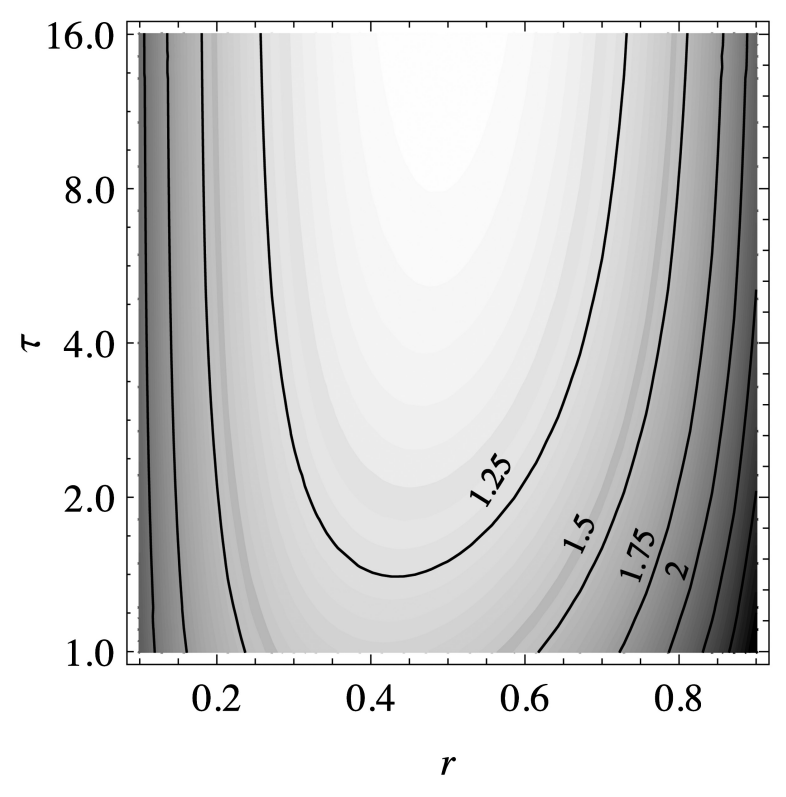

Figure A1: Plot of $Q_{\mathrm{fm}}(1+4 M)$, where $Q_{\mathrm{fm}}$ is approximated under the local diffusion limit and given by equation (A39) and where $1 /(1+4 M)$ is the local diffusion limit of $F_{\mathrm{ST}}$ under random mating and an even sex ratio. In this plot, $M=1$, although the qualitative pattern is independent of $M$. 


\section{Sex-Specific Migration}

When sex-specific migration is included, the probabilities of IBD $Q_{\mathrm{ff}}, Q_{\mathrm{fm}}$, and $Q_{\mathrm{mm}}$ take the following specific values:

$$
\begin{aligned}
Q_{\mathrm{ff}} & =\left(1-m_{\mathrm{f}}\right)^{2} Q^{\mathrm{J}}, \\
Q_{\mathrm{fm}} & =\left(1-m_{\mathrm{f}}\right)\left(1-m_{\mathrm{m}}\right) Q^{\mathrm{J}}, \\
Q_{\mathrm{mm}} & =\left(1-m_{\mathrm{m}}\right)^{2} Q^{\mathrm{J}},
\end{aligned}
$$

where $Q^{\mathrm{J}}$ is still given by equation (A36). Solving equations (A36) and (A40) yields

$$
\begin{aligned}
Q^{\mathrm{J}}=[N(r+\tau)-1] & \left(N(r+\tau)-1-2 N r \tau[N(1-r)-1] m_{\mathrm{f}}^{2}\right. \\
- & 2[N(1-r) \tau-1](N r-1) m_{\mathrm{m}}^{2}-\left[N(r+\tau)-1+4 N^{2} r(1-r) \tau\right] m_{\mathrm{f}} m_{\mathrm{m}} \\
+ & \{N(\tau+r)-1+4 N r \tau[2 N(1-r)-1]\} m_{\mathrm{f}} \\
- & \left.\{3(N(r+\tau)-1)-4 N r \tau[2 N(1-r)+1]\} m_{\mathrm{m}}\right)^{-1} .
\end{aligned}
$$

\section{Overlapping Generations}

In "Selection Gradients and Relatedness," we show that calculating $\Delta p$ under overlapping generations generates a relatedness coefficient $R_{a s, u v}$, given by equation (A28), that contains genetic identities of the form $\overline{p_{u \tilde{u} i j} p_{v \bar{v} i j}}$ for the

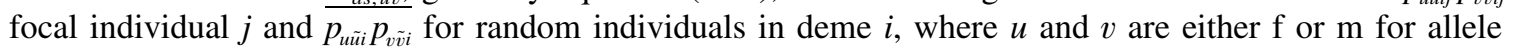
frequencies in females or males, respectively and $\tilde{u}$ and $\tilde{v}$ are either $\mathrm{f}$ or $\mathrm{m}$, denoting that the allele is maternally or paternally derived, respectively. The identity $\overline{p_{u i u j}^{2}}$ is simply the probability that an allele in individual $j$ of sex $u$ donated by a parent of sex $\tilde{u}$ is $A$, which is simply the frequency $p$. The remaining genetic identities in $R_{a s, u v}$ for alleles sampled in the focal individual, $\overline{p_{\mathrm{ff} i j} p_{\mathrm{fm} i j}}$ and $\overline{p_{\mathrm{m} i j} p_{\mathrm{mm} i j}}$, are both given by

$$
Q_{\mathrm{fm}} p+\left(1-Q_{\mathrm{fm}}\right) p^{2}=p^{2}+Q_{\mathrm{fm}} p q,
$$

which is just the probability that both alleles in the focal individual are $A$.

The identity $\overline{p_{\text {uüi }}^{2}}$, which is the probability that two alleles drawn from individuals of sex $u$ donated by parents of sex $\tilde{u}$ are both $A$, is given by

$$
\frac{1}{N_{u}} p+\left(1-\frac{1}{N_{u}}\right)\left(p^{2}+Q_{u[\tilde{u} u u[\tilde{u}]} p q\right)
$$

where $Q_{u[\tilde{u} u[\tilde{u}]}$ is the probability that two alleles drawn from different individuals of sex $u$ and donated by parents of sex $\tilde{u}$ are identical by descent. When the two alleles are drawn from individuals of the same sex but one is maternally derived and the other paternally derived, the genetic identity is

$$
\overline{p_{u f i} p_{u \mathrm{~m} i}}=\frac{1}{N_{u}}\left(p^{2}+Q_{\mathrm{fm}} p q\right)+\left(1-\frac{1}{N_{u}}\right)\left(p^{2}+Q_{u[\mathrm{f}] u[\mathrm{~m}]} p q\right) .
$$

Finally, when the alleles are drawn from different sexes, the identity is

$$
\overline{p_{\mathrm{fu} u} p_{\mathrm{m} \tilde{i} i}}=p^{2}+Q_{\mathrm{f}[\tilde{u} \mathrm{~m}[\tilde{v}]} p q .
$$

Calculating the IBD probabilities with overlapping generations requires taking into account that alleles can be sampled from surviving adults (who survive with probability $s_{u}$ if they are of sex $u$ ), juveniles who have just survived to the adult stage, or a mixture of both. Taking this into account for the probability that two alleles 
drawn from different individuals, one of $\operatorname{sex} u$ and the other of sex $v$, we find that the stationary value of this probability is given by

$$
\begin{aligned}
Q_{u v}=s_{u} s_{v} Q_{u v} & +\frac{s_{u}\left(1-s_{v}\right)(1-m)}{2}\left[\frac{1}{N_{u}} \frac{1+Q_{\mathrm{fm}}}{2}+\left(1-\frac{1}{N_{u}}\right) Q_{u u}+Q_{\mathrm{fm}}\right] \\
& +\frac{s_{v}\left(1-s_{u}\right)(1-m)}{2}\left[\frac{1}{N_{v}} \frac{1+Q_{\mathrm{fm}}}{2}+\left(1-\frac{1}{N_{v}}\right) Q_{v v}+Q_{\mathrm{fm}}\right]+\left(1-s_{u}\right)\left(1-s_{v}\right)(1-m)^{2} Q^{J},
\end{aligned}
$$

where $Q^{\mathrm{J}}$ is still given by equation (A36). We cannot specify the recursions for imprinted IBD probabilities with a single equation, since, as we saw above with the genetic identities, the equations will differ, depending on whether the alleles are sampled from individuals of the same sex and whether they are both maternally or paternally derived or one of each. The stationary probability that two alleles drawn from different individuals and donated by parents all of the same sex, $Q_{u[u] u[u]}$, is given by

$$
Q_{u[u] u[u]}=s_{u}^{2} Q_{u[u] u[u]}+2 s_{u}\left(1-s_{u}\right)(1-m)\left[\frac{1}{N_{u}} \frac{1+Q_{\mathrm{fm}}}{2}+\left(1-\frac{1}{N_{u}}\right) \frac{\left.Q_{u[u] u[u]}+Q_{u[u] u[u]}\right]}{2}\right]+\left(1-s_{u}\right)^{2}(1-m)^{2} Q_{u u}^{\mathrm{J}}
$$

and

$$
Q_{u u}^{\mathrm{J}}=C_{21 u} \frac{1+Q_{\mathrm{fm}}}{2}+\left(1-C_{21 u}\right) Q_{u u}
$$

When the sex of parents donating the sampled alleles is the same but different from the sex of the individuals sampled,

$$
Q_{u_{\hat{u}} u_{\hat{u}}}=s_{u}^{2} Q_{u_{\hat{u}} u_{\hat{u}}}+s_{u}\left(1-s_{u}\right)(1-m)\left(Q_{u_{\hat{i}} \hat{u}_{u}}+Q_{\mathrm{f}_{\hat{u}} \mathrm{~m}_{\hat{u}}}\right)+\left(1-s_{u}\right)^{2}(1-m)^{2} Q_{\hat{u} \hat{u}}^{\mathrm{J}} .
$$

The stationary probability that two alleles sampled from the same sex are donated by parents of different sexes is

$$
\begin{aligned}
Q_{u[\mathrm{f}] u[\mathrm{~m}]}= & s_{u}^{2} Q_{u[\mathrm{f}] u[\mathrm{~m}]}+s_{u}\left(1-s_{u}\right)(1-m) \\
& \times\left[\frac{1}{N_{u}} \frac{1+Q_{\mathrm{fm}}}{2}+\left(1-\frac{1}{N_{u}}\right) \frac{Q_{u[\mathrm{f}] u[\mathrm{~m}]}+Q_{u_{\hat{u}} \hat{u}_{\hat{u}}}}{2}+\frac{Q_{\mathrm{f}[\mathrm{f}] \mathrm{m}[\mathrm{m}]}+Q_{u_{u} \hat{u}_{u}}}{2}\right]+\left(1-s_{u}\right)^{2}(1-m)^{2} Q_{\mathrm{fm}} .
\end{aligned}
$$

When alleles are sampled from different sexes but donated by the same sex, the stationary probability is

$$
\begin{aligned}
Q_{\mathrm{f}[u] \mathrm{m}[u]}=s_{\mathrm{f}} s_{\mathrm{m}} Q_{\mathrm{f}[u] \mathrm{m}[u]} & +s_{u}\left(1-s_{\hat{u}}\right)(1-m)\left[\frac{1}{N_{u}} \frac{1+Q_{\mathrm{fm}}}{2}+\left(1-\frac{1}{N_{u}}\right) \frac{\left.Q_{u[u] u[u]}+Q_{u[\mathrm{f}] u[\mathrm{~m}]}\right]}{2}\right] \\
& +s_{\hat{u}}\left(1-s_{u}\right)(1-m) \frac{Q_{\mathrm{f}[u] \mathrm{m}[u]}+Q_{\mathrm{f}[\mathrm{m}] \mathrm{m}[\mathrm{f}]}}{2}+\left(1-s_{\mathrm{f}}\right)\left(1-s_{\mathrm{m}}\right)(1-m)^{2} Q_{u u}^{\mathrm{J}} .
\end{aligned}
$$

The last two stationary probabilities are for when the sexes of the donating parents and the sampled individuals are different: 
App. from J. Van Cleve et al., "Demography, Kinship, and Genomic Imprinting"

$$
\begin{aligned}
Q_{\mathrm{f}[\mathrm{f}] \mathrm{m}[\mathrm{m}]}=s_{\mathrm{f}} s_{\mathrm{m}} Q_{\mathrm{f}[\mathrm{f}] \mathrm{m}[\mathrm{m}]} & +s_{\mathrm{f}}\left(1-s_{\mathrm{m}}\right)(1-m) \frac{Q_{\mathrm{f}[\mathrm{f}] \mathrm{m}[\mathrm{f}]}+Q_{\mathrm{f}[\mathrm{f}] \mathrm{m}[\mathrm{m}]}}{2} \\
& +s_{\mathrm{m}}\left(1-s_{\mathrm{f}}\right)(1-m) \frac{Q_{\mathrm{f}[\mathrm{f}] \mathrm{m}[\mathrm{m}]}+Q_{\mathrm{f}[\mathrm{m}] \mathrm{m}[\mathrm{m}]}}{2}+\left(1-s_{\mathrm{f}}\right)\left(1-s_{\mathrm{m}}\right)(1-m)^{2} Q_{\mathrm{fm}}
\end{aligned}
$$

and

$$
\begin{aligned}
Q_{\mathrm{f}[\mathrm{m}] \mathrm{m}[\mathrm{f}]}=s_{\mathrm{f}} s_{\mathrm{m}} Q_{\mathrm{f}[\mathrm{f}] \mathrm{m}[\mathrm{m}]} & +s_{\mathrm{f}}\left(1-s_{\mathrm{m}}\right)(1-m)\left[\frac{1}{N_{\mathrm{f}}} \frac{1+Q_{\mathrm{fm}}}{2}+\left(1-\frac{1}{N_{\mathrm{f}}}\right) \frac{Q_{\mathrm{f}[\mathrm{f}] \mathrm{f}[\mathrm{m}]}+Q_{\mathrm{f}[\mathrm{m}] \mathrm{f}[\mathrm{m}]}}{2}\right] \\
& +s_{\mathrm{m}}\left(1-s_{\mathrm{f}}\right)(1-m)\left[\frac{1}{N_{\mathrm{m}}} \frac{1+Q_{\mathrm{fm}}}{2}+\left(1-\frac{1}{N_{\mathrm{m}}}\right) \frac{Q_{\mathrm{m}[\mathrm{f}] \mathrm{m}[\mathrm{f}]}+Q_{\mathrm{m}[\mathrm{f}] \mathrm{m}[\mathrm{m}]}}{2}\right] \\
& +\left(1-s_{\mathrm{f}}\right)\left(1-s_{\mathrm{m}}\right)(1-m)^{2} Q_{\mathrm{fm}} .
\end{aligned}
$$

Solving the equations given by expressions (A47)-(A53) yields all 10 imprinted IBD probabilities. The solutions to these equations were obtained in Mathematica and are available upon request.

\section{Dominance}

In order to include dominance, $h \neq 1 / 2$, in $\Delta p$, we need to calculate genetic identities that are three-element products of allele frequencies. It is straightforward to show that

$$
\overline{p_{\mathrm{f} i j}^{2} p_{\mathrm{m} i}}=\frac{1}{2} \overline{\left(p_{\mathrm{f} i j}+p_{\mathrm{ff} i j} p_{\mathrm{fm} i j}\right) p_{\mathrm{m} i}}=\frac{1}{2}\left(\overline{p_{\mathrm{f} i} p_{\mathrm{m} i}}+\overline{p_{\mathrm{ff} i j} p_{\mathrm{fm} i j} p_{\mathrm{f} i}}\right)
$$

To calculate $\overline{p_{\mathrm{ff} i j} p_{\mathrm{fm} i j} p_{\mathrm{f} i}}$, we need the probability of IBD of two homologous alleles taken from one adult and a third allele taken from a different adult, which we denote $Q_{32}$. Then,

$$
\begin{aligned}
\overline{p_{\mathrm{ff} i j} p_{\mathrm{fm} i j} p_{\mathrm{m} i}} & =Q_{32} p+3\left(Q_{\mathrm{fm}}-Q_{32}\right) p^{2}+\left(1-3 Q_{\mathrm{fm}}+2 Q_{32}\right) p^{3} \\
& =\overline{p_{\mathrm{mf} i j} p_{\mathrm{mm} i j} p_{\mathrm{f} i}} .
\end{aligned}
$$

Thus,

$$
\begin{aligned}
\overline{p_{\mathrm{f} i j}^{2} p_{\mathrm{m} i}} & =\frac{1}{2}\left[\left(Q_{\mathrm{fm}}+Q_{32}\right) p+\left(1+2 Q_{\mathrm{fm}}-3 Q_{32}\right) p^{2}+\left(13 Q_{\mathrm{fm}}+2 Q_{32}\right) p^{3}\right] \\
& =\overline{p_{\mathrm{f} i} p_{\mathrm{m} i j}^{2}}
\end{aligned}
$$

Let $Q_{33}$ represent the probability of IBD of three alleles drawn from three different adult individuals. We can write the remaining genetic identities as

$$
\overline{p_{\mathrm{f} i}^{2} p_{\mathrm{m} i}}=\frac{1}{N_{\mathrm{f}}}\left(p^{2}+Q_{\mathrm{fm}} p q\right)+\left(1-\frac{1}{N_{\mathrm{f}}}\right)\left[Q_{33} p+3\left(Q_{\mathrm{fm}}-Q_{33}\right) p^{2}+\left(1-3 Q_{\mathrm{fm}}+2 Q_{33}\right) p^{3}\right]
$$

and

$$
\overline{p_{\mathrm{f} i} p_{\mathrm{m} i}^{2}}=\frac{1}{N_{\mathrm{m}}}\left(p^{2}+Q_{\mathrm{fm}} p q\right)+\left(1-\frac{1}{N_{\mathrm{m}}}\right)\left[Q_{33} p+3\left(Q_{\mathrm{fm}}-Q_{33}\right) p^{2}+\left(1-3 Q_{\mathrm{fm}}+2 Q_{33}\right) p^{3}\right]
$$

Computing $Q_{33}$ requires the probability that three maternally derived alleles from three juveniles come from 
the same mother, $C_{\mathrm{f} 31}$, the probability that those alleles come from two females, $C_{\mathrm{f} 32}$, and analogous probabilities for paternally derived alleles, $C_{\mathrm{m} 31}$ and $C_{\mathrm{m} 32}$. These probabilities are

$$
\begin{aligned}
C_{\mathrm{f} 31}= & \frac{1}{N_{\mathrm{f}}^{2}}, \\
C_{\mathrm{f} 32}= & 3 \frac{1}{N_{\mathrm{f}}}\left(1-\frac{1}{N_{\mathrm{f}}}\right), \\
C_{\mathrm{m} 31}= & \frac{1}{N_{\mathrm{f}}^{2}}\left[\frac{1}{\tau^{2}}+\frac{3}{\tau}\left(1-\frac{1}{\tau}\right) \frac{1}{N_{\mathrm{m}}}+\left(1-\frac{1}{\tau}\right)\left(1-\frac{2}{\tau}\right) \frac{1}{N_{\mathrm{m}}^{2}}\right)+\frac{3}{N_{\mathrm{f}}}\left(1-\frac{1}{N_{\mathrm{f}}}\right)\left(\frac{1}{\tau}+\left(1-\frac{1}{\tau}\right) \frac{1}{N_{\mathrm{m}}}\right] \frac{1}{N_{\mathrm{m}}}+\left(1-\frac{1}{N_{\mathrm{f}}}\right)\left(1-\frac{2}{N_{\mathrm{f}}}\right) \frac{1}{N_{\mathrm{m}}^{2}}, \\
C_{\mathrm{m} 32}= & 3\left(\frac{1}{N_{\mathrm{f}}^{2}}\left[\frac{1}{\tau}\left(1-\frac{1}{\tau}\right)\left(1-\frac{1}{N_{\mathrm{m}}}\right)+\left(1-\frac{1}{\tau}\right)\left(1-\frac{2}{\tau}\right) \frac{1}{N_{\mathrm{m}}}\left(1-\frac{1}{N_{\mathrm{m}}}\right)\right]\right. \\
& +\frac{1}{N_{\mathrm{f}}}\left(1-\frac{1}{N_{\mathrm{f}}}\right)\left[\left(\frac{1}{\tau}\left(1-\frac{1}{N_{\mathrm{m}}}\right)+\left(1-\frac{1}{\tau}\right) \frac{3}{N_{\mathrm{m}}}\left(1-\frac{1}{N_{\mathrm{m}}}\right)\right]+\left(1-\frac{1}{N_{\mathrm{f}}}\right)\left(1-\frac{2}{N_{\mathrm{f}}}\right) \frac{1}{N_{\mathrm{m}}}\left(1-\frac{1}{N_{\mathrm{m}}}\right)\right\} .
\end{aligned}
$$

Then the probability that three alleles sampled from three different juvenile individuals are IBD is

$$
\begin{aligned}
Q_{33}^{\mathrm{J}}= & \frac{1}{8}\left[C_{\mathrm{f} 31} \frac{1+3 Q_{\mathrm{fm}}}{4}+\frac{C_{\mathrm{f} 32}}{2}\left(Q_{\mathrm{ff}}+Q_{(\mathrm{ffff})}\right)+\left(1-C_{\mathrm{f} 31}-C_{\mathrm{f} 32}\right) Q_{\mathrm{fff}}\right] \\
& +\frac{3}{8}\left[\frac{C_{\mathrm{f} 21}}{2}\left(Q_{\mathrm{fm}}+Q_{(\mathrm{ff}) \mathrm{m}}\right)+\left(1-C_{\mathrm{f} 21}\right) Q_{\mathrm{ffm}}\right] \\
& +\frac{3}{8}\left[\frac{C_{\mathrm{m} 21}}{2}\left(Q_{\mathrm{fm}}+Q_{(\mathrm{mm}) \mathrm{f}}\right)+\left(1-C_{\mathrm{m} 21}\right) Q_{\mathrm{fmm}}\right] \\
& +\frac{1}{8}\left[C_{\mathrm{m} 31} \frac{1+3 Q_{\mathrm{fm}}}{4}+\frac{C_{\mathrm{m} 32}}{2}\left(Q_{\mathrm{mm}}+Q_{(\mathrm{mm}) \mathrm{m}}\right)+\left(1-C_{\mathrm{m} 31}-C_{\mathrm{m} 32}\right) Q_{\mathrm{mmm}}\right],
\end{aligned}
$$

where $Q_{u v w}$ is the IBD probability for three alleles from three different adult individuals of sexes $u, v$, and $w$; $Q_{(u u) v}$ is the IBD probability for two homologous alleles sampled from an adult individual of sex $u$ and an allele from a different adult of sex $v$. Without sex-specific migration or generation overlap, $Q_{\mathrm{fff}}=Q_{\mathrm{ffm}}=Q_{\mathrm{fmm}}=$ $Q_{\mathrm{mmm}}=Q_{33}=(1-m)^{3} Q_{33}^{\mathrm{J}}$ and $Q_{(\mathrm{ff}) \mathrm{f}}=Q_{(\mathrm{ff}) \mathrm{m}}=Q_{(\mathrm{mm}) \mathrm{f}}=Q_{(\mathrm{mm}) \mathrm{m}}=Q_{32}=(1-m)^{2} Q_{32}^{\mathrm{J}}$. This final IBD probability is

$$
Q_{32}^{\mathrm{J}}=\frac{1}{2}\left[\frac{C_{\mathrm{f} 21}}{2}\left(Q_{\mathrm{fm}}+Q_{(\mathrm{ff}) \mathrm{m}}\right)+\left(1-C_{\mathrm{f} 21}\right) Q_{\mathrm{ffm}}+\frac{C_{\mathrm{m} 21}}{2}\left(Q_{\mathrm{fm}}+Q_{(\mathrm{mm}) \mathrm{f}}\right)+\left(1-C_{\mathrm{m} 21}\right) Q_{\mathrm{fmm}}\right] .
$$

The probabilities $Q_{33}$ and $Q_{32}$ can be computed by substituting them appropriately into equations (A63) and (A64) and solving the resulting equations simultaneously.

\section{Sex-Specific Migration and Change in Frequency of $A$}

In this section, we prove condition (20), which says that under sex-specific migration and no multiple paternity, the direction of viability selection alone determines which allele is silenced; the silenced allele is donated by the sex experiencing stronger viability selection. Our method entails assuming that $\mathcal{S}_{x}\left(z_{a a}^{x}\right)=0$ and determining whether $\mathcal{S}_{y}\left(z_{a a}^{x}\right)$ is greater or less than 0 . As long as the fertility cost $C$ and survival benefits $B_{\mathrm{f}}$ and $B_{\mathrm{m}}$ change monotonically near $z_{a a}^{x}$, then $z_{a a}^{y}$ will be greater than $z_{a a}^{x}$ when $\mathcal{S}_{y}\left(z_{a a}^{x}\right)>0$ and $z_{a a}^{y}<z_{a a}^{x}$ when $\mathcal{S}_{y}\left(z_{a a}^{x}\right)<0$. 
Assume that $B_{\mathrm{f}}>B_{\mathrm{m}}$. Combining $\mathcal{S}_{x}\left(z_{a a}^{x}\right)=0$ with $\mathcal{S}_{y}\left(z_{a a}^{x}\right)$, we find that

$$
\mathcal{S}_{y}\left(z_{a a}^{x}\right) \propto-\left(B_{\mathrm{m}}-C\right) R_{j s, \mathrm{~mm}}\left(1-\frac{R_{j s, \mathrm{mf}} R_{j s, \mathrm{fm}}}{R_{j s, \mathrm{ff}} R_{j s, \mathrm{~mm}}}\right) .
$$

As long as $N \geq 4$, the constants $R_{j s, u v}$ defined in equation (19) are nonnegative. This means that for $\mathcal{S}_{x}\left(z_{a a}^{x}\right)=0$ to hold, equation (18) shows that $B_{\mathrm{f}}-C>0$ and $B_{\mathrm{m}}-C<0$. Further, we can show that

$$
R_{j s, \mathrm{mf}} R_{j s, \mathrm{fm}}-R_{j s, \mathrm{ff}} R_{j s, \mathrm{~mm}} \propto 2 N(N-2)\left(1+3 Q_{\mathrm{fm}}\right)\left(2-m_{\mathrm{f}}-m_{\mathrm{m}}\right)\left(m_{\mathrm{f}}-m_{\mathrm{m}}\right)^{2}>0
$$

which means that $\mathcal{S}_{y}\left(z_{a a}^{x}\right)>0$ and $z_{a a}^{y}>z_{a a}^{x}$. Similar reasoning leads to $z_{a a}^{y}<z_{a a}^{x}$ when $B_{\mathrm{f}}<B_{\mathrm{m}}$ and yields

$$
\left(z_{a a}^{y}-z_{a a}^{x}\right)\left(B_{\mathrm{f}}-B_{\mathrm{m}}\right)>0,
$$

which simplifies to condition (20) for determining whether $z_{a a}^{y}-z_{a a}^{x}>0$ is true.

\section{Overlapping Generations}

In this section, we prove inequality (23). First, assume that $\tau=1$, that $m_{\mathrm{f}}=m_{\mathrm{m}}$, and that the effect of the mutant allele on survival is the same for both sexes and for both adults and juveniles and is given by $B$. Then, the relatedness coefficients $R_{b, \mathrm{ff}}=R_{b, \mathrm{mf}}=R_{j s, \mathrm{ff}}=R_{j s, \mathrm{mf}}=R_{j s, \mathrm{f}}$ and $R_{b, \mathrm{fm}}=R_{b, \mathrm{~mm}}=R_{j s, \mathrm{fm}}=R_{j s, \mathrm{~mm}}=R_{j s, \mathrm{~m}}$ in the selection gradients in equations (7) and (8), respectively. The expression for $\Delta p$ from equation (6) then becomes, to first order in $\delta$,

$$
\begin{aligned}
& \Delta p=p q\{[\left.B\left(\alpha_{\mathrm{f}} s_{\mathrm{f}} R_{a s, \mathrm{ff}}+\alpha_{\mathrm{m}} s_{\mathrm{m}} R_{a s, \mathrm{mf}}\right)+2 \beta(B-C) R_{j s, \mathrm{f}}\right] \delta_{x} \\
&\left.+\left[B\left(\alpha_{\mathrm{f}} s_{\mathrm{f}} R_{a s, \mathrm{fm}}+\alpha_{\mathrm{m}} s_{\mathrm{m}} R_{a s, \mathrm{~mm}}\right)+2 \beta(B-C) R_{j s, \mathrm{~m}}\right] \delta_{y}\right\},
\end{aligned}
$$

where the relatedness coefficients $R_{a s, u v}$ are given by equation (22). Following the method in "Sex-Specific Migration and Change in Frequency of $A$," we obtain $\mathcal{S}_{x}\left(z_{a a}\right)$ and $\mathcal{S}_{y}\left(z_{a a}\right)$ from equation (A68), set $\mathcal{S}_{x}\left(z_{a a}^{x}\right)=0$, and determine the sign of $\mathcal{S}_{y}\left(z_{a a}^{x}\right)$. After using $\mathcal{S}_{x}\left(z_{a a}^{x}\right)=0$ to solve for $C$ and plugging the result into $\mathcal{S}_{y}\left(z_{a a}\right)$, we find that

$$
\mathcal{S}_{y}\left(z_{a a}^{x}\right) \propto B\left[\alpha_{\mathrm{f}} s_{\mathrm{f}} R_{a s, \mathrm{fm}}+\alpha_{\mathrm{m}} s_{\mathrm{m}} R_{a s, \mathrm{~mm}}+2 \beta R_{j s, \mathrm{~m}}-\frac{R_{j s, \mathrm{~m}}}{R_{j s, \mathrm{f}}}\left(\alpha_{\mathrm{f}} s_{\mathrm{f}} R_{a s, \mathrm{ff}}+\alpha_{\mathrm{m}} s_{\mathrm{m}} R_{a s, \mathrm{mf}}+2 \beta R_{j s, \mathrm{f}}\right)\right] .
$$

Since gene expression has a positive effect of survival $(B>0)$ and given that $\mathcal{S}_{y}\left(z_{a a}^{x}\right)>0$ implies $z_{a a}^{y}>z_{a a}^{x}$ and vice versa, we can rearrange equation (A69) to yield

$$
\left(z_{a a}^{y}-z_{a a}^{x}\right)\left(\frac{\alpha_{\mathrm{f}} s_{\mathrm{f}} R_{a s, \mathrm{fm}}+\alpha_{\mathrm{m}} s_{\mathrm{m}} R_{a s, \mathrm{~mm}}}{R_{j s, \mathrm{~m}}}-\frac{\alpha_{\mathrm{f}} s_{\mathrm{f}} R_{a s, \mathrm{ff}}+\alpha_{\mathrm{m}} s_{\mathrm{m}} R_{a s, \mathrm{mf}}}{R_{j s, \mathrm{f}}}\right)>0,
$$

which simplifies to inequality (23) for determining whether $z_{a a}^{y}-z_{a a}^{x}>0$ is true.

\section{The Effect of Dominance on $\Delta p$}

In our analysis of $\Delta p$ in equation (6), we noted that allelic dominance itself is not likely to select for imprinting but rather may affect the strength of selection for imprinting. An additional effect of dominance is that it adds frequency dependence to the sign of $\Delta p$. To show this, we can substitute the genetic identities in equation (6) for their equivalent expressions in terms of IBD probabilities and allele frequencies; these expressions are derived in "Probabilities of Identity by Descent." The resulting expression for $\Delta p$ is too bulky to present here, but it shows that

$$
\Delta p \propto p q\left\{(1-h)\left[G_{x}\left(z_{a a}\right) \delta_{x}+G_{y}\left(z_{a a}\right) \delta_{y}\right]+(1-2 h) H\left(z_{a a}, p\right)\left(\delta_{x}+\delta_{y}\right)\right\}+O\left(\delta^{2}\right)
$$


where $G_{x}\left(z_{a a}\right)$ and $G_{y}\left(z_{a a}\right)$ are functions of $z_{a a}$ and $H\left(z_{a a}, p\right)$ is a function of both $z_{a a}$ and $p$. Generically, dominance adds frequency dependence to the sign of the change in allele frequency, since over- and underdominance can create stable and unstable polymorphisms, respectively. Dominance can also affect the strength of selection for an allele in a frequency-dependent manner by masking or unmasking its effect in heterozygotes. It is this latter effect that we study here by assuming that $1 \leq h \leq 0$.

Since our analysis of the additive case showed that multiple paternity selects for silencing of the maternally derived copy of the resource-enhancing gene independent of the demographic parameters of the model, we use a local diffusion limit $(N \rightarrow \infty, m \rightarrow 0$, and $N m \rightarrow M)$ to obtain an intuition for the effect of dominance and frequency dependence on $\Delta p$.

First, when $M$ is large, local drift within demes is weak, and we find that

$$
\Delta p=\frac{p q}{4}\left((1-h)\left[\left(B_{\mathrm{f}}+B_{\mathrm{m}}-2 C\right) \delta_{x}+\left(B_{\mathrm{f}}+B_{\mathrm{m}}-\frac{2}{\tau} C\right) \delta_{y}\right]-p(1-2 h)\left\{B_{\mathrm{f}}+B_{\mathrm{m}}-\left[2-\left(1-\frac{1}{\tau}\right)\right] C\right\}\left(\delta_{x}+\delta_{y}\right)\right),
$$

where we neglect terms of order $1 / M$ and $\delta^{2}$. Equation (A71) demonstrates that for large local deme sizes, dominance of $A(h<1 / 2)$ reduces the fertility cost and survival benefit of resource-enhancing gene expression. This effect is amplified as the frequency of $A$ increases. More importantly, increases in $\tau$ yield decreases in $\mathcal{S}_{x}$ and increases in $\mathcal{S}_{y}$, which means that $z_{a a}^{y}$ increases and $z_{a a}^{x}$ decreases. In effect, this implies stronger selection for imprinting as the effect of the resident allele is partially masked for $h<1 / 2$. When $a$ is dominant $(h>1 / 2)$, the effect is reversed. This reasoning suggests that for any value of $p$, the ratio $z_{a a}^{x} / z_{a a}^{y}$ increases as $h$ increases. Since $\mathcal{S}_{x}=\mathcal{S}_{x}$ when $h=1$ and the mutant allele is recessive, $z_{a a}^{x} / z_{a a}^{y}<1$ for all values of $h$ and $p$, which suggests that silencing of expression from the maternally derived allele still evolves under multiple paternity.

When $M$ is small, local drift within demes is strong relative to the effect of migration. In this case,

$$
\Delta p=\frac{2 M p q r(1-r)}{1+(r / \tau)}\left[\left(B_{\mathrm{f}}+B_{\mathrm{m}}-\frac{2}{\tau} C\right) \delta_{y}-(1-2 h) C\left(1-\frac{1}{\tau}\right)\left(\delta_{x}-\delta_{y}\right)\right]
$$

neglecting terms of order $M^{2}$ and $\delta^{2}$. In contrast to the case when $M$ is large, equation (A72) shows that strong local drift eliminates the frequency dependence induced by dominance. Nevertheless, the effect of dominance is still the same, namely, to increase $z_{a a}^{x} / z_{a a}^{y}$ as $h$ increases. In sum, analysis of the diffusion limit of $\Delta p$ suggests that dominance does not change the prediction that multiple paternity selects for silencing of the maternally derived copy of a resource-enhancing gene. Rather, increasing $h$ brings $z_{a a}^{x}$ closer to $z_{a a}^{y}$ and decreases the strength of selection for imprinted gene expression.

\section{Literature Cited Only in the Appendix}

Rice, J. A. 1995. Mathematical statistics and data analysis. 2nd ed. Duxbury, Belmont, CA.

Roze, D., and F. Rousset. 2003. Selection and drift in subdivided populations: a straightforward method for deriving diffusion approximations and applications involving dominance, selfing and local extinctions. Genetics 165:2153-2166.

Wakeley, J. 2003. Polymorphism and divergence for island-model species. Genetics 163:411-420.

Wang, J. 1997. Effective size and $F$-statistics of subdivided populations. II. Dioecious species. Genetics 146: $1465-1474$. 